\title{
OPEN Pharmacoinformatics-based investigation of bioactive compounds of Rasam (South Indian recipe) against human cancer
}

\author{
Arjun Kumar Kalimuthu ${ }^{1}$, Theivendren Panneerselvam², Parasuraman Pavadai ${ }^{3}$, \\ Sureshbabu Ram Kumar Pandian ${ }^{1}$, Krishnan Sundar ${ }^{1}$, Sankaranarayanan Murugesan ${ }^{4}$, \\ Damodar Nayak Ammunje ${ }^{5}$, Sattanathan Kumar ${ }^{6}$, Sankarganesh Arunachalam ${ }^{1 \bowtie} \&$ \\ Selvaraj Kunjiappan ${ }^{1 \otimes}$
}

Spice-rich recipes are referred to as "functional foods" because they include a variety of bioactive chemicals that have health-promoting properties, in addition to their nutritional value. Using pharmacoinformatics-based analysis, we explored the relevance of bioactive chemicals found in Rasam (a South Indian cuisine) against oxidative stress-induced human malignancies. The Rasam is composed of twelve main ingredients, each of which contains a variety of bioactive chemicals. Sixtysix bioactive compounds were found from these ingredients, and their structures were downloaded from Pubchem. To find the right target via graph theoretical analysis (mitogen-activated protein kinase 6 (MAPK6)) and decipher their signaling route, a network was built. Sixty-six bioactive compounds were used for in silico molecular docking study against MAPK6 and compared with known MAPK6 inhibitor drug (PD-173955). The top four compounds were chosen for further study based on their docking scores and binding energies. In silico analysis predicted ADMET and physicochemical properties of the selected compounds and were used to assess their drug-likeness. Molecular dynamics (MD) simulation modelling methodology was also used to analyse the effectiveness and safety profile of selected bioactive chemicals based on the docking score, as well as to assess the stability of the MAPK6-ligand complex. Surprisingly, the discovered docking scores against MAPK6 revealed that the selected bioactive chemicals exhibit varying binding ability ranges between -3.5 and $-10.6 \mathrm{kcal} \mathrm{mol}^{-1}$. MD simulation validated the stability of four chemicals at the MAPK6 binding pockets, including Assafoetidinol A (ASA), Naringin (NAR), Rutin (RUT), and Tomatine (TOM). According to the results obtained, fifty of the sixty-six compounds showed higher binding energy $\left(-6.1\right.$ to $\left.-10.6 \mathrm{kcal} \mathrm{mol}^{-1}\right)$, and four of these compounds may be used as lead compounds to protect cells against oxidative stressinduced human malignancies.

Cancer is defined as unregulated cell or tissue growth that may spread to other parts of the body. It is the second greatest cause of mortality in the world, behind cardiovascular illnesses, and the number of cases continues to rise $^{1}$. According to the GLOBOCON-2020 report, there are around 19.30 million new cancer cases diagnosed and 10.00 million cancer deaths worldwide ${ }^{2}$. A range of modifiable health behaviours, such as high fat and simple carbohydrate diet, irregular eating habits as well as poor physical activity contributes to the sudden rise in cancer incidences ${ }^{3}$. Several studies have shown that dysregulated nutrition and sedentary life style are key factors in the cellular redox process, resulting in unwanted by-products such as reactive oxygen species (ROS),

${ }^{1}$ Department of Biotechnology, Kalasalingam Academy of Research and Education, Krishnankoil, Tamil Nadu 626126, India. 'Department of Pharmaceutical Chemistry, Swamy Vivekanandha College of Pharmacy, Elayampalayam, Tiruchengodu, Tamil Nadu 637205, India. ${ }^{3}$ Department of Pharmaceutical Chemistry, Faculty of Pharmacy, M.S. Ramaiah University of Applied Sciences, M S R Nagar, Bengaluru, Karnataka 560054, India. ${ }^{4}$ Department of Pharmacy, Birla Institute of Technology and Science Pilani, Pilani Campus, Vidya Vihar, Pilani, Rajasthan 333031, India. ${ }^{5}$ Department of Pharmacology, Faculty of Pharmacy, M.S. Ramaiah University of Applied Sciences, M S R Nagar, Bengaluru, Karnataka 560054, India. ${ }^{6}$ Deparment of Pharmaceutical Chemistry, Paavai College of Pharmacy and Research, Namakkal, Tamil Nadu 637018, India. ${ }^{\circledR}$ email: sankarganesh@ gmail.com; selvaraj.k@klu.ac.in 
reactive nitrogen species (RNS), and DNA reactive aldehyde ${ }^{4-6}$. In mitochondria, ROS is an unavoidable byproduct of oxidative phosphorylation ${ }^{7}$. ROS is a two-edged sword that has both helpful (at low concentration) and harmful (at high concentration) properties. At low concentration, ROS regulates cellular activities such as cell cycle, proliferation, differentiation, migration, and death while an increased quantity of ROS may damage proteins, nucleic acids, lipids, membranes, and organelles, it also reduces cell viability and causes apoptosis ${ }^{8,9}$.

The production of reactive oxygen species (ROS) in cells is neutralized by a number of antioxidant defense mechanisms. The detoxifying enzymes and antioxidant enzymes protect cells and tissues from toxins and oxidative stress ${ }^{10}$. Oxidative stress sensitive genes accomplish ROS scavenging by secreting antioxidant enzymes including superoxide dismutase (SOD), catalase, glutathione peroxidase, peroxiredoxins, and other non-enzymatic compounds such as flavonoids, carotenoids, glutathione, $\alpha$-lipoic acid, iron chelators, vitamins $\mathrm{A}, \mathrm{C}$ and $\mathrm{E}^{11}$. Furthermore, increased levels of intracellular ROS beyond a certain threshold cause down regulation of cellular antioxidant pathways and enzyme systems, resulting in malignant transformation via various molecular targets such as nuclear factor-B (NF-B), nuclear factor E2 (erythroid-derived 2)-related factor 2 (Nrf2), Kelch likeECH-associated protein 1 (Keap1), mitogen-activated protein kinases (MAPKs) and phosphoinositide 3-kinase $(\mathrm{PI} 3 \mathrm{~K})^{12}$.

The MAPKs are a class of serine/threonine protein kinases that play important roles in controlling extracellular signaling into a wide range of cellular processes ${ }^{13}$. Based on their structure and functions, they are classified into conventional and atypical MAPKs. Conventional MAPKs, such as, extracellular signal-regulated kinase (ERK) $1 / 2$ and p38 isoforms ( $\alpha, \beta, \gamma$, and $\delta$ ), and atypical MAPKs are ERK3/4 and ERK7/8 ${ }^{14}$. The ERK $1 / 2$ and p38 MAPK pathways have been targeted by numerous drugs to battle the various types of cancer with some clinical success. While compared with ERK 1/2 (conventional) MAPKs, much less work has been explored on ERK3, also known as MAPK6 signaling, and its cellular functions ${ }^{15}$. The ERK3 has significant physiological functions, including pulmonary differentiation, $\mathrm{T}$ cell activation, and angiogenesis ${ }^{16}$. In addition, MAPK6 has been connecting a series of signaling cascades and play a major role in the migration and invasiveness of certain types of cancers. MAPK6 is essential for production of several cellular factors including interleukin-8 (IL-8), in both, normal and tumorigenic cells ${ }^{16}$. MAPK6 is a widely expressed protein in all tissues with highest expression levels detected in skeletal muscle, brain, and gastrointestinal tract. MAPK6 interacts with and phosphorylates steroid receptor coactivator 3 (SRC-3), an oncogenic protein overexpressed in multiple human cancers at the amino acid residue, serine $857(\mathrm{~S} 857)^{17}$.

Spices are used in cuisine all around the globe for their taste, flavour and their health advantages ${ }^{18}$. Because, they contain numerous bioactive components, certain spices have been utilized in Indian traditional medicine to prevent and cure numerous ailments, including cancer $^{19}$. Capsaicin (red pepper) ${ }^{1}$, curcumin (turmeric) $)^{20}$, piperine (black pepper) ${ }^{21}$, lycopene (tomato) ${ }^{22}$, myricetin (tea) ${ }^{23}$, and rutin (buckwheat) ${ }^{24}$ are a few examples of bioactive chemicals that have been shown to possess antioxidant and anticancer properties. "Rasam" is a famous South Indian spicy soup that has been made fresh every day and served with rice ${ }^{25}$. Tamarind, red pepper, black pepper, cumin seed, fenugreek, asafoetida, garlic, tomato, coriander, curry leaves, sesame oil, and mustard are the main flavors (spices) of Rasam ${ }^{26}$. These spices that used to make Rasam, a "functional food", include a plethora of bioactive chemicals that have been linked to improved tumor prognosis ${ }^{27}$. The synergistic activity of a mixed bioactive chemicals is always greater than that of a single component ${ }^{28}$. Furthermore, these bioactive chemicals function via many signaling pathways and display anticancer activity by blocking certain signaling cascades that drive unregulated cell division and proliferation ${ }^{29}$. Bioactive substances may also inhibit the malignant transformation by targeting pro-tumorigenic cells or the pro-metabolic carcinogen's conversion ${ }^{30}$.

Cancer cells acquire resistance to cancer treatments by several mechanisms, importantly mutated genes, proteins, enzymes and transcription factors. These mutated genes, enzymes, proteins and transcription factors are thought to be significant drug targets for slowing down the progression of cancer ${ }^{31}$. The signaling networks, such as genes, proteins, and enzymes, are shown in this perspective using graph theoretical network analysis. The graph theoretical concepts applied in the multifaceted signaling network, it may be possible to systematically examine the topology and functions of these selected network. Further, the graph theoretical network analysis can be used to predict the structural and dynamic properties of selected signaling pathway. Such predictions can assist the selection of ideal drug targets for reducing the complications of cancer ${ }^{27}$. It also gives data on the active site and molecular interactions of bioactive active compounds (ligands), which may help in the molecular docking study. As a result, the current research used pharmacoinformatics to examine the relevance of bioactive chemicals found in Rasam spices against oxidative stress-induced human malignancies. The predicted ADMET (absorption, distribution, metabolism, excretion, and toxicity) characteristics of the selected bioactive compounds were also investigated. Further, molecular dynamics simulation was investigated to determine the stability and binding modes of selected bioactive compounds with an appropriate cancer receptor protein.

\section{Materials and methods}

Graph theoretical network analysis. The graph theoretical network analysis was built using Cytoscape software version 3.7.1 and the Kyoto Encyclopedia of Genes and Genomes (KEGG) database ${ }^{28}$. The functions of numerous genes and proteins involved in the MAPK6/ERK3 (ko04657) in Homo sapiens was chosen to recognize the influential proteins.

Protein preparation. The RCSB Protein Data Bank (PDB: http://www.rcsb.org/pdb) provided the X-ray crystallographic structure of MAPK6 (PDB ID: 7AQB) ${ }^{32}$. Prior to analysis, the protein was cleaned and missing residues were inserted using Swiss-PDB Viewer v4.1.0. The file was named target.pdb and saved for further analysis. We also utilized BIOVIA Discovery Studio Visualizer version 4.0 software (Accelrys Software Inc., San 
Diego, CA) in order to determine the protein structure and amino acid position from active regions, which was then utilized for molecular docking study.

Active compounds retrieval and preparation. We found that around sixty-six bioactive components from twelve spices were used to make Rasam. The identified sixty-six components along with one known MAPK6 inhibitor drug were collected using the data repository (Indian Medicinal Plants, Phytochemistry, and Therapeutics (IMPPAT)) (https://cb.imsc.res.in/imppat/home), previously published studies ${ }^{33}$ and public database PubChem (https://pubchem.ncbi.nlm.nih.gov/).

Binding site identification. A binding site in the target is a particular location on an enzyme/protein that permits the enzyme to attach to certain molecules and perform a chemical reaction. The major strategy to treat a disease is the binding of ligands or bioactive chemicals to the specific location of a protein/enzyme. This helps the bioactive chemicals to create enough contact sites in order to establish robust interaction with target enzymes by ensuring optimal and favourable catalytic areas. Using the Prank Web (https://prankweb.cz/) server, all possible active binding sites of targeted compounds were found for further analysis. Using the PyRx program, a receptor grid was created once the active site of the protein was selected.

Molecular docking. Molecular docking approach is a crucial component of structural biology research, and it is one among the widely used technique in the process of drug design. The PyRx 0.8 tool $^{34}$ and AutoDock Vina program $^{35}$ was used to accomplish the molecular docking study. The ligand was one of the selected bioactive chemicals, and the receptor was MAPK6 (PDB ID: 7AQB). Polar hydrogen atoms and Kollman partial charges were introduced into the $3 \mathrm{D}$ structure using PyRx software. To compute docking energy affinities $\left(\mathrm{kcal} \mathrm{mol}^{-1}\right)$, the receptor and ligand files were stored in ".pdbqt" format. For each ligand, AutoDock Vina calculated the energy affinity values of up to ten different docking positions. AutoDock Vina effects were used to calculate each complex affinity energies based on the ligand conformation at the active binding site with RMSD between the original and subsequent structures taken into consideration. The amount of hydrogen bonds and non-covalent interactions for each complex were calculated using Discovery Studio Visualizer, which produced details, compounds, and interaction pictures $(2 \mathrm{D} \text { and } 3 \mathrm{D})^{36}$.

Prediction of in silico pharmacokinetic and physicochemical properties. In silico prediction of ADME and physicochemical properties of the selected bioactive chemicals plays a major role in determining its integrity and efficiency. Selected bioactive chemicals into account, properties like molecular weight, molar refractivity, solubility, bioavailability, bioavailability radar plot, egg-boiled model, brain penetration, and human gastro intestinal absorption properties of the active bio-compounds have been determined using SwissADME (http://www.swissadme.ch/) webserver. The SwissADME webserver is a free tool that can predict the pharmacokinetic and drug-likeness properties of the test bioactive compounds ${ }^{37,38}$.

Toxicity prediction. Toxicity was predicted by determining the safety profile of the intended bioactive chemicals, which must have deadly effects on people and cause organ damage. As a result, the toxicity of the chosen bioactive chemicals was assessed using pkCSM-pharmacokinetics web-based server (http://biosig.unime lb.edu.au/pkcsm/prediction) ${ }^{39}$.

Molecular dynamics simulation. The molecular dynamic simulation was evaluated to determine the binding stability, conformation and interaction modes between the selected bioactive compounds (ligands) and receptor (MAPK6). The selected ligand-MAPK6 complex files were subjected to molecular dynamics studies using GROMACS 2019.2 software ${ }^{40-42}$. The selected ligands topology was downloaded from PRODRG server ${ }^{43}$. The system preparation of all the complexes were as described earlier ${ }^{24}$. For molecular dynamic simulation, first vacuum was minimized using the steepest descent algorithm for 5000 steps. The complex structure was solvated in a cubic periodic box of $0.5 \mathrm{~nm}$ with a simple point charge (SPC) water model. The complex system was subsequently maintained with an appropriate salt concentration of $0.15 \mathrm{M}$ by adding a suitable amount of $\mathrm{Na}^{+}$and $\mathrm{Cl}^{-}$counter ions. Each complex was allowed a simulation time of $50 \mathrm{~ns}$ from the NPT (Isothermal-Isobaric, constant number of particles, pressure, and temperature) equilibration was subjected in NPT ensemble for final run. The trajectory analysis of root means square deviation (RMSD) and root mean square fluctuation (RMSF) was performed in the GROMACS simulation package through the online server "WebGRO for Macromolecular Simulations (https://simlab.uams.edu/)".

Molecular mechanics Poisson-Boltzmann surface area (MMPBSA) calculation. The MMPBSA method was used to calculate the protein-ligand binding free energy of each complex. The free energy of binding was determined using the g_mmpbsa tool developed for GROMACS ${ }^{24,44}$.

Consent to participate. All authors agree to participate.

Consent for publication. All authors agree for publication. 


\begin{abstract}
Results
Graph theoretical network analysis. A graph was constructed for the MAPK6/ERK3 signaling pathway network. Numerous entities of genes, proteins (nodes) and their interactions (edges) in the current research work was displayed in Fig. 1. Based on centrality criteria such as degree, proximity, eccentricity, eigen vector, and radiality, the network has 92 nodes and 153 edges. The measured values of Betweennes (3483. 24) degree (2), closeness (0.004), eccentricity (0.125), eigen vector (0.0538), radiality $(10.08)$, and stress $(20,850)$ have shown the threshold value of all measures as well as significant node in the network (Tables 1,2). The protein mitogen activated protein kinase 6 (MAPK6)/extracellular signal-regulated kinase 3 (ERK3) was identified as a potential drug target based on the centrality measure and its threshold values.
\end{abstract}

Retrieval of bioactive compounds and preparation. The accessible bioactive components of the selected spices (tamarind, red pepper, black pepper, cumin seed, fenugreek, asafoetida, garlic, tomato, coriander, curry leaves, sesame oil, and mustard) were searched using IMPPAT database. From the database, a list of sixtysix bioactive compounds were obtained from the twelve spices along with one standard MAPK6 inhibitor listed in Table 3.

Binding site identification. Our evaluation of the crystal structure of MAPK6 (PDB: 7AQB) revealed the existence of 11 binding pockets, according to binding site analyses. The protein's recovered binding site residue are shown in the Fig. 2. Molecular docking investigations were also conducted using the obtained complex structure of the binding sites. Grid generation in molecular docking resulted in more reliable ligand posture scoring. As a result, we created a receptor grid for the selected MAPK6 protein based on the previously acquired binding site residues to achieve more precise scoring of our ligand poses. A receptor grid with a box dimensions of $\mathrm{X}=38.6666, \mathrm{Y}=62.5914$, and $\mathrm{Z}=31.9740$ in angstrom $(\AA)$ was created and utilized further for molecular docking experiments.

Molecular docking. The optimum intermolecular interaction between the target protein and bioactive chemicals were investigated using molecular docking analysis. To analyse their binding capability, a specific number of bioactive chemicals (sixty-six) and known MAPK6 inhibitor were docked against MAPK6 using AutoDock Vina. Twelve bioactive chemicals were shown to have a lower binding energy $\left(<-9 \mathrm{kcal} \mathrm{mol}^{-1}\right)$ with the target protein. The binding energy of the bioactive compounds following molecular docking was found to be scattered, ranging from -3.50 to $-10.60 \mathrm{kcal} \mathrm{mol}^{-1}$, as illustrated in Fig. 3 and Table 3 . The top four compounds (Assafoetidinol A $\left(-9.80 \mathrm{kcal} \mathrm{mol}^{-1}\right)$, Naringin $\left(-9.60 \mathrm{kcal} \mathrm{mol}^{-1}\right)$, Rutin $\left(-9.80 \mathrm{kcal} \mathrm{mol}^{-1}\right)$, Tomatine $\left.\left(-10.60 \mathrm{kcal} \mathrm{mol}^{-1}\right)\right)$ were chosen for future research based on their binding energy with the amino acid residues in the active site of MAPK6. In this study, we used a standard MAPK6 inhibitor (CID: 447077) (-9.30 kcal mol ${ }^{-1}$ ) as control, due to previously reported inhibitory activity against Bcr-Abl-dependent cell growth with an $\mathrm{IC}_{50}$ of 2-35 $\mathrm{nM}$ in different cell lines ${ }^{45}$.

Interpretation of protein-ligand interactions. The interactions formed between the selected four ligands and MAPK6 has been visualized using BIOVIA Discovery studio visualizer tool. It was observed that compound CID: 12041593 (ASA) showed better interaction with MAPK6 and the binding energy was $9.80 \mathrm{kcal} \mathrm{mol}^{-1}$. Compound CID: 12041593 formed four Van der Waals interactions with TYR110A (3.81 Å), GLU120 (3.57 $\AA$ ), GLU163A (3.74 ̊) and TRP196B (3.86 ̊), two conventional and two carbon hydrogen bonds TYR110A (2.09 $\AA)$, ALA156B (2.10 ̊) and GLU112A (3.58 ̊), ASN161A (2.85 ̊) respectively. Alkyl and PiAlkyl bond was also found at the position ARG45A (3.64 ̊), ALA116B (3.75 $\AA$ ), and PRO115B (3.67 ̊), respectively showed in Fig. 4 and Table 4. For the compound CID: 442428 (NAR) it has been observed four hydrophobic and four hydrogen bonds with desired active site amino acid residues of MAPK6. CID: 442428 hydrophobic interactions with ALA116B (3.84 $⿱$ ), LYS154B (3.76 $)$, GLU163A (3.86 $⿱$ ) and THR194B (3.75 $)$ ), and four

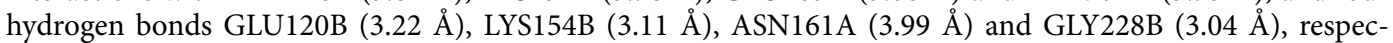
tively showed in Fig. 5 and Table 4. In the case of CID: 5280805 (RUT) exhibited four hydrophobic bonds

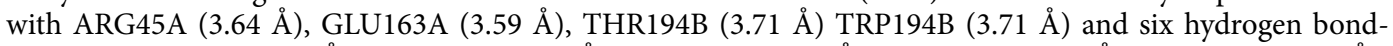

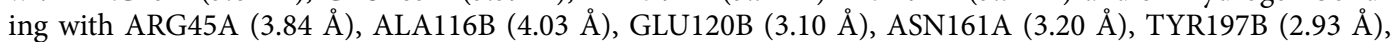
GLU224B (3.44 $\AA$ ) between active site amino acid residues of desired protein MAPK6, depicted in Fig. 6 and Table 4. For, CID: 28523 (TOM), it has observed two hydrophobic bonding with TYR134A (3.97 Å), VAL166A (3.75 $\AA$ ), and four hydrogen bonding with GLY32B (3.42 $\AA$ ), ARG45A (3.25 $)$ ), ASN161A (4.08 $)$, THR230B $(3.25 \AA$ ) between the target protein, and showed in Fig. 7 and Table 4. For the standard MAPK6 inhibitor drug (CID: 447077) was observed five hydrophobic interactions with TYR110A (3.49 ̊), LYS154B (3.67 ̊), GLU163

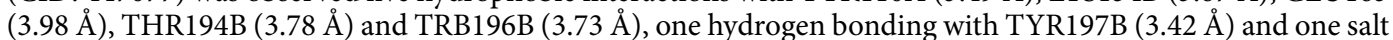
bridge GLU224B (3.68 $\AA$ ) between the target protein, and showed in Fig. 8 and Table 4.

Pharmacokinetic and physicochemical properties prediction analysis. The ADME and physicochemical properties of selected bioactive compounds from Rasam were assessed through SwissADME (http:// www.swissadme.ch/) webserver and these are presented in Table 5. From the assessed data in Table 5, the compound ASA (CID: 12041593; molecular weight $398.49 \mathrm{~g} \mathrm{~mol}^{-1}$ ) is alone found not to violate Lipinski's rule of five. Other three compounds NAR (CID: 442428; molecular weight $580.53 \mathrm{~g} \mathrm{~mol}^{-1}$ ), RUT (CID: 5280805; molecular weight $610.52 \mathrm{~g} \mathrm{~mol}^{-1}$ ), TOM (CID: 28523; molecular weight $1431.59 \mathrm{~g} \mathrm{~mol}^{-1}$ ) and standard drug PD-173955 (molecular weight $443.35 \mathrm{~g} \mathrm{~mol}^{-1}$ ) violated Lipinski's rule of five, due to higher molecular weight. The polar surface area of selected compounds was $79.90 \AA^{2}$ (CID: 12041593), 225.06 $\AA^{2}$ (CID: 442428), 269.43 $\AA^{2}$ (CID: 


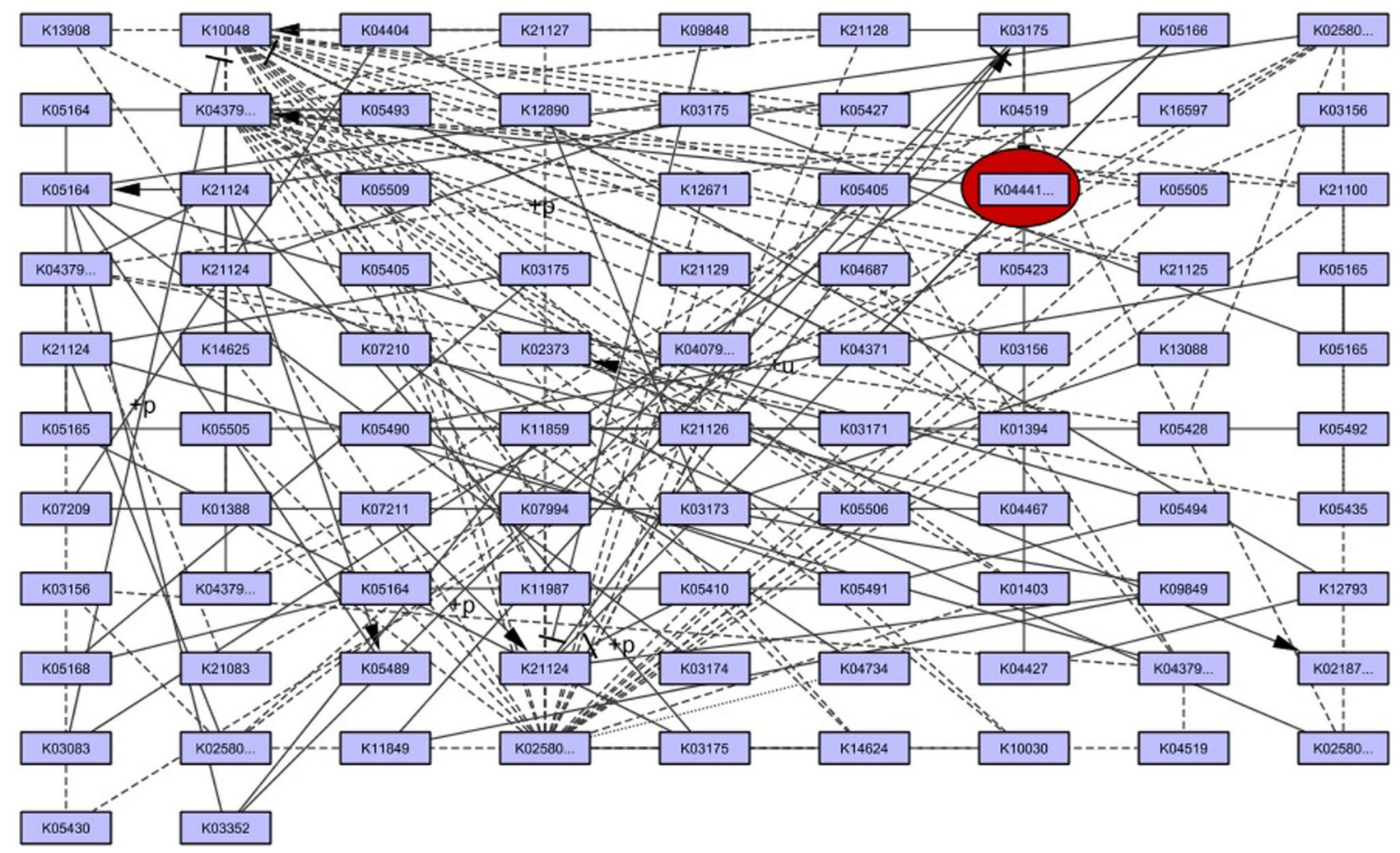

Figure 1. The signaling pathway of MAPK6/ERK3.

\begin{tabular}{|l|l|r|l|}
\hline Centrality measures & Maximum & \multicolumn{1}{l|}{ Mean } & Minimum \\
\hline Betweenness & 3483.24 & 467.19 & 13.34 \\
\hline Closeness & 0.004 & 0.0017 & 0.0013 \\
\hline Degree & 27 & 2.985 & 2 \\
\hline Eigenvector & 0.0538 & 0.0247 & 0.0000000001 \\
\hline Eccentricity & 0.125 & 0.0564 & 0.0625 \\
\hline Radiality & 10.08 & 5.37 & 4.59 \\
\hline Stress & 20,850 & 4529.82 & 114 \\
\hline Nodes & 92 & & \\
\hline Edges & 153 & & \\
\hline
\end{tabular}

Table 1. The nodes and edges of influential protein (MAPK6).

5280805), 308.40 $\AA^{2}$ (CID: 28523) and 85.11 $\AA^{2}$. The observed results also demonstrated that CID: 12041593 and standard drug PD-173955 (CID: 447077) possesses a better human gastro intestinal (GI) absorption property, CID: 442428, CID: 5280805 and CID: 28523 have lower GI absorption properties. In general, higher GI absorption leads to enhanced bioavailability of the bioactive compound (CID: 12041593) of food (Rasam). Therefore, it might be better absorbed from the gastrointestinal tract upon oral administration. The higher number of $\mathrm{H}$-bonds are possibly measured to be involved during protein ligand binding. From the result, the bioavailability score of four compounds showed better results (+0.55 for CID: 12041593 and standard MAPK6 inhibitor PD-173955, and + 0.17 for other three compounds) thereby relating with molecular properties CID: 12041593. Of the four compounds analyzed only ASA (CID: 12041593) and standard MAPK6 inhibitor (PD-173955) was predicted to have better chances as a possible drug-relevant candidate with anticancer potential. All the four bioactive compounds are soluble in nature, except standard drug (CID: 447077). The solubility class of all the compounds are listed in the Table 5. The synthetic accessibility score was found to be > 6 except CID: 12041593 and the standard drug (CID: 447077), which indicated that other three compounds are very difficult to synthesize.

The graphical representation of drug-likeness of four selected compounds are presented in the Fig. 9. The pink area within the hexagon represents the optimal range for the compounds. The recommended range for drug-like compound was insaturation (INSATU): fraction of carbons in the sp3 hybridization not less than 0.25, insolubility (INSOLU): $\log S$ not higher than 6, hydrophobicity (LIPO): between -0.7 and +5.0 , rotatable bonds (FLEXI): 


\begin{tabular}{|c|c|c|c|c|c|c|c|}
\hline Gene & Betweenness & Closeness & Degree & Eccentricity & EIGENVECTOR & Radiality & Stress \\
\hline $\begin{array}{l}\text { K04441, K04440, K04371, K06855, } \\
\text { K04464, K19603 }\end{array}$ & 52.33876592 & 0.003717 & 2 & 0.090909 & 0.053801959 & 9.747253 & 144 \\
\hline K07209, & 103.6985941 & 0.002174 & 2 & 0.071429 & $8.95 \mathrm{E}-05$ & 7.648352 & 156 \\
\hline K04427 & 471.5981179 & 0.003413 & 2 & 0.083333 & 0.006866011 & 9.483516 & 720 \\
\hline K04467 & 63.85047619 & 0.002137 & 2 & 0.0625 & $6.64 \mathrm{E}-04$ & 7.56044 & 114 \\
\hline K03175 & 2009.804357 & 0.003922 & 6 & 0.090909 & 0.058181247 & 10.08791 & 14,254 \\
\hline K05405 & 13.34193734 & 0.003106 & 3 & 0.076923 & 0.142419691 & 9.351648 & 1088 \\
\hline K12793 & 334.5981179 & 0.002833 & 2 & 0.076923 & $8.11 \mathrm{E}-04$ & 8.824176 & 486 \\
\hline K04404 & 196.8481179 & 0.002398 & 2 & 0.071429 & $1.05 \mathrm{E}-04$ & 8.120879 & 298 \\
\hline K04734 & 253.4018821 & 0.002604 & 2 & 0.071429 & 0.04760016 & 8.483516 & 8984 \\
\hline K07210 & 116.4018821 & 0.002179 & 2 & 0.0625 & 0.005617381 & 7.659341 & 494 \\
\hline K02580, K04735, & 560.0648877 & 0.002959 & 25 & 0.071429 & 0.403361797 & 9.175824 & 18,072 \\
\hline $\begin{array}{l}\text { K04379, K09029, K04448, K04449, } \\
\text { K04502 }\end{array}$ & 218.7644444 & 0.003333 & 25 & 0.076923 & 0.40408361 & 9.593407 & 792 \\
\hline K05494 & 1928.456998 & 0.003175 & 2 & 0.125 & $2.93 \mathrm{E}-04$ & 9.241758 & 18,144 \\
\hline K05491 & 1918.882826 & 0.002747 & 3 & 0.111111 & $3.51 \mathrm{E}-05$ & 8.703297 & 17,860 \\
\hline K03175 & 773.1666667 & 0.002597 & 2 & 0.090909 & $4.05 \mathrm{E}-05$ & 8.659341 & 4284 \\
\hline K21124 & 1667.40129 & 0.003077 & 5 & 0.1 & $3.43 \mathrm{E}-04$ & 9.318681 & 13,242 \\
\hline K21124 & 896.4314357 & 0.003534 & 6 & \begin{tabular}{|l|l|}
0.1 & \\
\end{tabular} & 0.008856044 & 9.78022 & 2316 \\
\hline K05164 & 3483.249418 & 0.003623 & 6 & 0.125 & 0.002486075 & 9.857143 & 32,586 \\
\hline K05166 & 1803.658925 & 0.004065 & 4 & 0.111111 & 0.012947182 & 10 & 20,850 \\
\hline K09849 & 457.7 & 0.003012 & 3 & \begin{tabular}{|l|l|}
0.090909 \\
\end{tabular} & 0.001870239 & 9.054945 & 1648 \\
\hline K03173 & 308 & 0.002551 & 3 & \begin{tabular}{|l|}
0.076923 \\
\end{tabular} & $2.24 \mathrm{E}-04$ & 8.395604 & 1098 \\
\hline K10048 & 2756.21084 & 0.003745 & 27 & \begin{tabular}{|l|}
0.083333 \\
\end{tabular} & 0.416220404 & 9.769231 & 34,478 \\
\hline K03083 & 610.4522186 & 0.003497 & 2 & 0.1 & 0.049949843 & 9.56044 & 10,184 \\
\hline K04371 & 610.4522186 & 0.003472 & 2 & \begin{tabular}{|l|}
0.090909 \\
\end{tabular} & 0.049949843 & 9.538462 & 10,184 \\
\hline K03352 & 1252.614593 & 0.003636 & 3 & \begin{tabular}{|l|}
0.1 \\
\end{tabular} & 0.007157894 & 9.681319 & 11,160 \\
\hline K11849 & 145.1490196 & 0.003215 & 2 & 0.083333 & 0.006989256 & 9.285714 & 856 \\
\hline
\end{tabular}

Table 2. The results of threshold parameter values of the MAPK6 network analysis.

no more than 9 rotatable bonds, molecular weight (SIZE): between 150 and $500 \mathrm{~g} \mathrm{~mol}^{-1}$ and polar surface area (POLAR): between 20 and $130 \AA^{2}$ ). The compound CID: 12041593 (ASA) possess drug-like properties and are displayed by the red slanted hexagon within the pink shade (Fig. 9). Other three compounds (CID: 442428, CID: 5280805 and CID: 28523) and standard drug (CID: 447077) have high polar surface area, poor solubility and high molecular weight (size) which indicated off-shoot of the vertex (polar and size).

Moreover, the pharmacokinetic properties were investigated by using egg-boiled model of selected four compounds and standard MAPK6 inhibitor is depicted in Fig. 10. The egg-boiled model is helpful to predict two key pharmacokinetic properties simultaneously, i.e., the passive gastrointestinal absorption and blood brain barrier (BBB) penetration. The egg-shaped organisation plot shows that the compound present in the yolk (i.e. yellow region) represent the highly probable $\mathrm{BBB}$ permeation whereas albumin (i.e. white region) represent the highly probable human intestinal absorption. From the Fig. 10, the compound ASA (CID: 12041593) and standard drug (CID: 447077) found in albumin (white region) elucidated the good absorption in gastrointestinal region. Remaining three compounds were found to be outside of the boiled-egg region. From the above observed results, it can be interpreted that the compound ASA (CID: 12041593) have sufficient potential to be drug.

Analysis of toxicity. In silico toxicity prediction of the selected four compounds has been performed using pkCSM-pharmacokinetics web-based server. The server has identified drug-induced hERG toxicity, AMES toxicity, $\mathrm{LD}_{50}$, hepatotoxicity, skin sensitization, Tetrahymena pyriformis (TP) toxicity, and minnow toxicity which was listed in Table 6.

Molecular dynamics simulation. Although, protein-ligand docking was widespread and has successful application, it just gives the static view of the binding pose of ligand in the active site of the receptor similar to a photographic image. Molecular dynamics (MD) must be employed to simulate the dynamics of atoms in the system as a function of time with integration of Newton's equations of motions ${ }^{46}$. MD simulations for 50 ns were carried out for the top four receptor-ligand complexes obtained from the docking studies, that is 7AQB-ASA, 7AQB-NAR, 7AQB-RUT, 7AQB-TOM, 7AQB-STD and unbound apo form of the target MAPK6 protein (PDB ID: 7AQB) and their results were interpreted. To decipher the stability and fluctuations of these complexes, MD trajectories analysis was performed with the help of RMSD (Root Mean Square Deviation), RMSF (Root Mean Square Fluctuation), RG (Radius of gyration) and SASA (Solvent Accessible Surface Area) of receptor atoms. 


\begin{tabular}{|c|c|c|c|c|}
\hline S. No & Ingredients & Compound id (CID) & Bioactive compound & Docking score $\left(\mathrm{kcal} \mathrm{mol}^{-1}\right)$ \\
\hline 1 & \multirow{9}{*}{ Turmeric } & 969516 & Curcumin & -7.8 \\
\hline 2 & & 5469424 & Demethoxycurcumin & -7 \\
\hline 3 & & 5315472 & Bisdemethoxycurcumin & -7.7 \\
\hline 4 & & 10250249 & 5'-methoxycurcumin & -6.7 \\
\hline 5 & & 443160 & (+)-Alpha-Phellandrene & -6.6 \\
\hline 6 & & 10429233 & Dihydrocurcumin & -7.2 \\
\hline 7 & & 92776 & Zingiberene & -6.2 \\
\hline 8 & & 10887971 & $(+)$-Sabinene & -6.6 \\
\hline 9 & & 64685 & Borneol & -5.2 \\
\hline 10 & \multirow{6}{*}{ Red pepper } & 1548943 & Capsaicin & -6.1 \\
\hline 11 & & 5281229 & Capsorubin & -8 \\
\hline 12 & & 5281228 & Capsanthin & -8.4 \\
\hline 13 & & \begin{tabular}{|l|l|}
77994099 \\
\end{tabular} & Bicyclomahanimbicine & -9.5 \\
\hline 14 & & 107982 & Dihydrocapsaicin & -6.2 \\
\hline 15 & & 448438 & Violaxanthin & -9.4 \\
\hline 16 & \multirow{6}{*}{ Asafoetida } & 131751454 & Assafoetidin & -8.3 \\
\hline 17 & & 7067262 & Franesiferol A & -7.3 \\
\hline 18 & & 15559239 & Franesiferol C & -8.9 \\
\hline 19 & & 11892267 & Conferol & -9.2 \\
\hline 20 & & 12041593 & Assafoetidinol A & -9.8 \\
\hline 21 & & 636584 & Assafoetidinol B & -9.5 \\
\hline 22 & \multirow{5}{*}{ Cumin } & 95779 & Thymohydroquinone & -6 \\
\hline 23 & & 637563 & $t$-anethole & -6.1 \\
\hline 24 & & 6989 & Thymol & -6.8 \\
\hline 25 & & 5282799 & Dihomolinoleic acid & -5.2 \\
\hline 26 & & 985 & Palmitic acid & -5.1 \\
\hline 27 & \multirow{11}{*}{ Mustard } & 370 & Gallic acid & -5.8 \\
\hline 28 & & 5280343 & Quercetin & -8.1 \\
\hline 29 & & 5280805 & Rutin & -9.8 \\
\hline 30 & & 689043 & Caffeic acid & -6.4 \\
\hline 31 & & 445858 & Ferulic acid & -6.5 \\
\hline 32 & & 637542 & $p$-Coumaric acid & -6.6 \\
\hline 33 & & 1183 & Vanillin gallic acid & -6.1 \\
\hline 34 & & 65064 & (-)-Epigallocatechin Gallate & -8.9 \\
\hline 35 & & 107905 & (-)-Epicatechin Gallate & -9.5 \\
\hline 36 & & 442428 & Naringin C & -9.6 \\
\hline 37 & & 5281855 & Ellagic Acid & -8.3 \\
\hline 38 & \multirow{6}{*}{ Sesame oil } & 101746 & Sesamolin & -9.4 \\
\hline 39 & & 94672 & Sesaminol & -9.5 \\
\hline 40 & & 5281235 & Beta-Cryptoxanthin & -8.7 \\
\hline 41 & & 135404715 & Hydroxysesamone & -7.2 \\
\hline 42 & & 360837 & 2,3-epoxysesamone & -7.8 \\
\hline 43 & & 72307 & Sesamin & -9.4 \\
\hline 44 & \multirow{6}{*}{ Black pepper } & 971 & Oxalic acid & -3.8 \\
\hline 45 & & 10364 & Carvacrol & -6.5 \\
\hline 46 & & 5318825 & Koenigine & -8.2 \\
\hline 47 & & 278055 & Koenigicine & -7.7 \\
\hline 48 & & 59053143 & Murrayastine & -7.5 \\
\hline 49 & & 398941 & Dithymoquinone & -3.3 \\
\hline 50 & \multirow{4}{*}{ Coriander } & 10282 & Monoterpene & -6.3 \\
\hline 51 & & 6549 & Linalool & -4.8 \\
\hline 52 & & 1549026 & Geranyl acetate & -5.3 \\
\hline 53 & & 6654 & $\alpha$-pinene & -5.5 \\
\hline 54 & Tamarind & 875 & Tartaric acid & -5.1 \\
\hline Contir & & & & \\
\hline
\end{tabular}




\begin{tabular}{|c|c|c|c|c|}
\hline S. No & Ingredients & Compound id (CID) & Bioactive compound & Docking score $\left(\mathrm{kcal} \mathrm{mol}^{-1}\right)$ \\
\hline 55 & \multirow{5}{*}{ Garlic } & 65036 & Allicin & -4.4 \\
\hline 56 & & 16590 & Diallyl disulfide & -3.5 \\
\hline 57 & & 16315 & Diallyl trisulfide & -3.6 \\
\hline 58 & & 5386591 & Ajoene & -4.2 \\
\hline 59 & & 9793905 & S-allyl-cysteine & -4.4 \\
\hline 60 & \multirow{4}{*}{ Fenugreek } & 5570 & Trigonelline & -5.5 \\
\hline 61 & & 354616 & Gentianine & -6.1 \\
\hline 62 & & 444170 & Fenugreekine & -8.5 \\
\hline 63 & & 5280441 & Vitexin & -8.9 \\
\hline 64 & \multirow{3}{*}{ Tomato } & 446925 & Lycopene & -7.4 \\
\hline 65 & & 28523 & Tomatine & -10.6 \\
\hline 66 & & 54670067 & Ascorbic acid & -5.7 \\
\hline 67 & Standard MAPK6 inhibitor & 447077 & $\begin{array}{l}\text { PD-173955 (6-(2,6-dichlorophenyl)-8-methyl-2-\{[3-methylsulfanyl)phenyl] amino\}- } \\
\text { 7H,8H-pyrido[2,3-d]pyrimidin-7-one) }\end{array}$ & -9.3 \\
\hline
\end{tabular}

Table 3. Bioactive compounds in Rasam ingredients and their binding affinity against MAPK6.

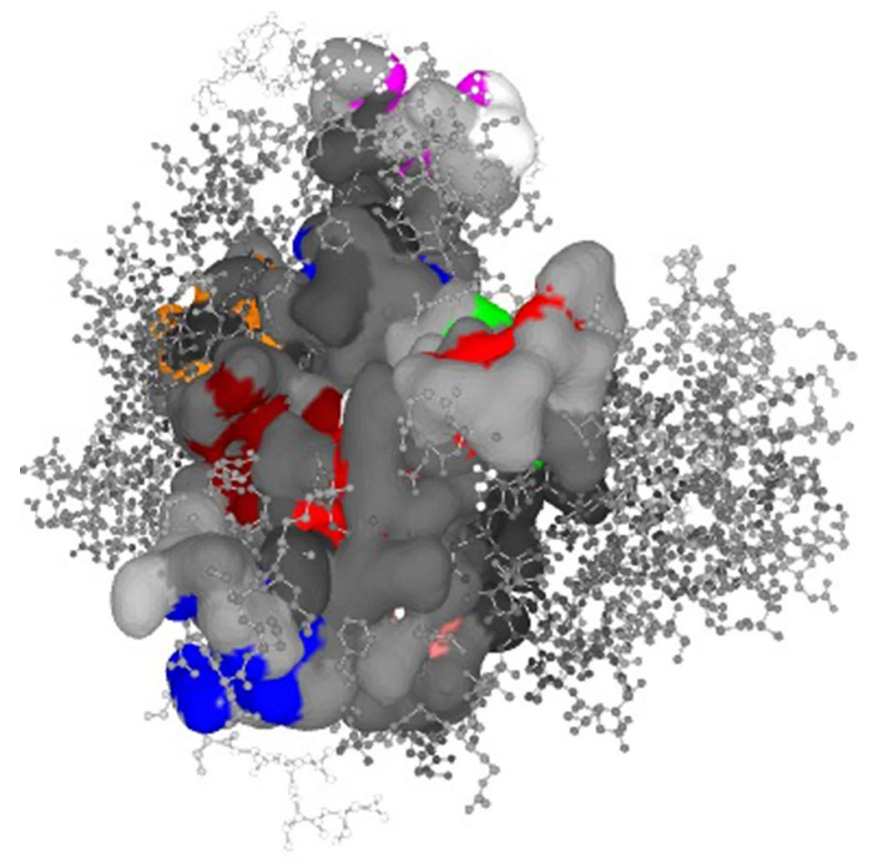

Figure 2. Showing active site and correspondence binding site of MAPK6 protein. Eleven binding pockets were predicted with different colour. Binding pocket 1 (17 AA with predicted score 9.16), pocket 2 (14 AA with predicted score 8.01), pocket 3 (15 AA with predicted score 4.75), pocket 4 (12 AA with predicted score 3.43 ), pocket 5 (13 AA with predicted score 3.10), pocket 6 (11 AA with predicted score 3.0), pocket 7 (10 AA with predicted score 2.14), pocket 8 (12 AA with predicted score 2.04), pocket 9 (08 AA with predicted score 1.90), pocket 10 (11 AA with predicted score 1.77) and pocket 11 (08 AA with predicted score 1.75).

RMSD is an important parameter to analyse the equilibration of MD trajectories and check the stability of complex systems during the simulation process. RMSD of the protein backbone atoms were plotted against time to assess its variations in structural conformation. Initially, the 7AQB-ASA complex showed variations in backbone RMSD till $30 \mathrm{~ns}$ ranging from 0.15 to $0.44 \mathrm{~nm}$. The stable conformation was attained in the time period between 21 and $50 \mathrm{~ns}$ with no considerable deviations in the values (Fig. 11). 7AQB-NAR complex showed variations in backbone RMSD till $20 \mathrm{~ns}$ ranging from 0.17 to $0.43 \mathrm{~nm}$. The stable conformation was attained in the time period between 21 and 50 ns with no considerable deviations in the values (Fig. 11). The 7AQB-RUT complex showed variations in backbone RMSD till $30 \mathrm{~ns}$ ranging from 0.13 to $0.35 \mathrm{~nm}$. The stable conformation was attained in the time period between 31-50 ns with no considerable deviations in the values (Fig. 8). The 7AQB-TOM complex showed variations in backbone RMSD till $35 \mathrm{~ns}$ ranging from 0.14 to $0.43 \mathrm{~nm}$. The first stable conformation was attained in the time period between 36 and $50 \mathrm{~ns}$ with no considerable deviations in the 


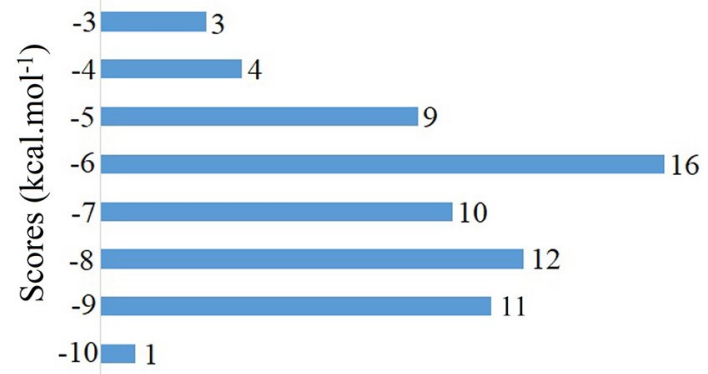

Number of compounds

Figure 3. Showing the range of docking score distribution of sixty-six phytochemicals presence in the Rasam.
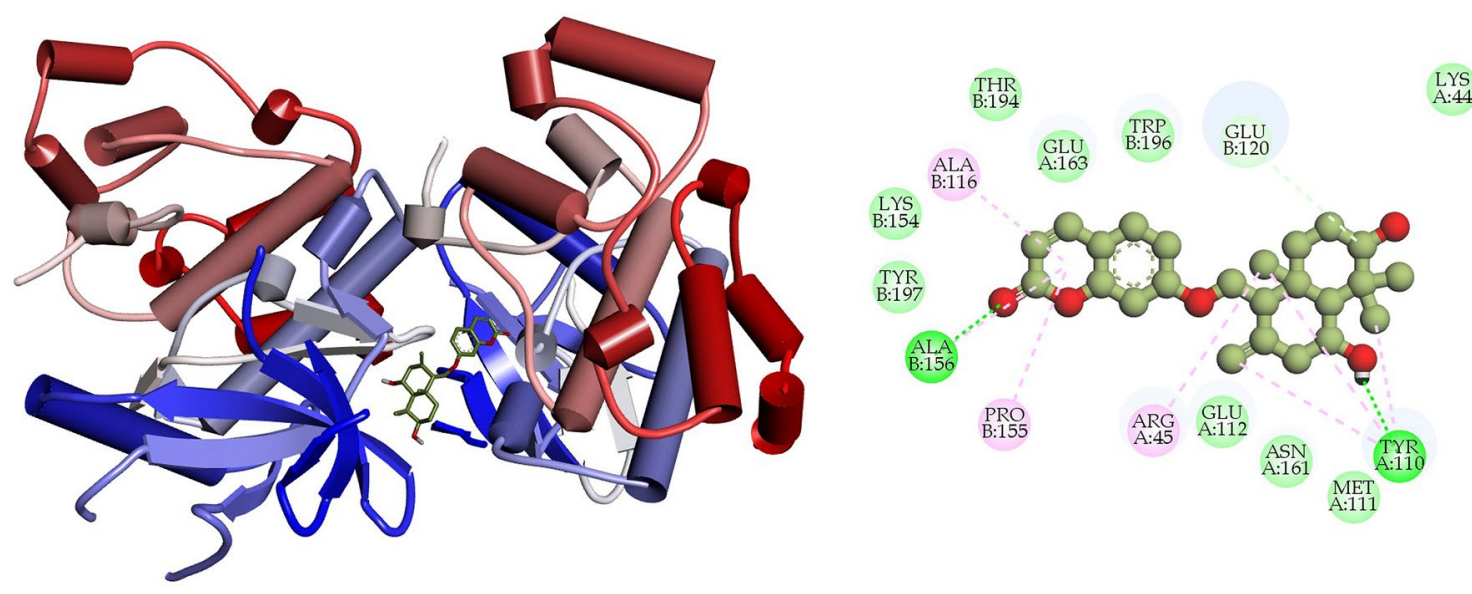

Figure 4. Depicted the interaction between the compound CID: 12041593 (Assafoetidinol A) and MAPK6. Left side representing $3 \mathrm{D}$ and the right side representing $2 \mathrm{D}$ complex protein-ligand interaction.

values (Fig. 11). This clearly specifies that the protein underwent small structural changes in all the complexes during simulations.

RMSF is an another crucial parameter while examining the stability and flexibility of complex systems during simulation ${ }^{47}$. RMSF was examined to analyse the changes in the behaviour of amino acid residues of target protein on binding to a ligand ${ }^{48,49}$. The RMSF values for C $\boldsymbol{\alpha}$ atoms of the protein were calculated and plotted with respect to the residues. In all the complexes, examined, the amino acid residues showed minimal fluctuations throughout the simulation. The amino acids of MAPK6 which interacted with ASA during docking showed minimal fluctuation values during MD simulation viz. CYS28, GLY29, LYS185 and LYS229, with NAR it showed low fluctuation values during MD simulation viz GLY29 and LEU192, with RUT showed minimal fluctuation values during MD simulation viz. GLY29, ARG70, LYS229 and ASN269 and with TOM it showed moderate fluctuation values during MD simulation viz. GLY29, LYS185, SER189, TYR266 and PRO301 (Fig. 12). These results revealed that binding of the ligands actuated no major effects on the flexibility of the protein.

Further, Radius of gyration $(\mathrm{Rg})$ of the complex systems were also analysed. Rg is the root mean square distance of the atoms of the protein from the axis of rotation ${ }^{49}$. It is one among the important parameter that represents the overall change in the protein structure compactness and its dimensions during the simulation ${ }^{50}$. Higher Rg values characterize the protein as less compact and flexible while low values depict the high compactness and rigidity $^{47}$. Rg values of backbone atoms of protein were plotted against time to examine the changes in structural compactness. Binding of ASA decreased the backbone Rg values till $30 \mathrm{~ns}$. In the time period between 31 and $50 \mathrm{~ns}$ there were no considerable fluctuations and almost constant value of $\sim 1.98 \mathrm{~nm}$ was maintained. Till end, the $\mathrm{Rg}$ values were found to be in the range between 1.95 and $1.99 \mathrm{~nm}$. Complete analysis revealed that, in the initial stage the trajectory had shown its peak value of $2.12 \mathrm{~nm}$. Later this high value was never displayed again which shows the stability of protein in the complex (Fig. 13). Binding of NAR decreased the backbone Rg values till $15 \mathrm{~ns}$. In the time period between 16 and $45 \mathrm{~ns}$ there were no considerable fluctuations and almost constant value of $\sim 2.04 \mathrm{~nm}$ was maintained. Till end, the $\mathrm{Rg}$ values were found to be in the range of $2.02-2.05 \mathrm{~nm}$. Complete analysis revealed that, in the initial stage, the trajectory showed its peak value of $\sim 2.09 \mathrm{~nm}$. Later, this high value was never displayed again which shows the stability of protein in the complex (Fig. 13). Binding of RUT decreased the backbone Rg values till $31 \mathrm{~ns}$. In the time period between 32 and 50 ns there were no considerable fluctuations and almost constant value of $\sim 2.03 \mathrm{~nm}$ was maintained. Till end, the $\mathrm{Rg}$ values were found to be in the range of 2.00-2.05 $\mathrm{nm}$. Complete analysis revealed that, in the initial stage, the trajectory exhibited its peak value of $\sim 2.10 \mathrm{~nm}$. Later, this high value was never displayed again which shows the stability of protein in the 


\begin{tabular}{|c|c|c|c|c|}
\hline Compounds & Residues & \begin{tabular}{|l|} 
Amino \\
Acid
\end{tabular} & Distance $(\AA)$ & Bond category \\
\hline \multirow{12}{*}{ CID: 12041593 Assafoetidinol A } & $110 \mathrm{~A}$ & TYR & 3.81 & Hydrophobic \\
\hline & $120 \mathrm{~B}$ & GLU & 3.57 & Hydrophobic \\
\hline & $163 \mathrm{~A}$ & GLU & 3.74 & Hydrophobic \\
\hline & 196B & TRP & 3.86 & Hydrophobic \\
\hline & $110 \mathrm{~A}$ & TYR & 2.09 & Hydrogen \\
\hline & $112 \mathrm{~A}$ & GLU & 3.58 & Hydrogen \\
\hline & $156 \mathrm{~B}$ & ALA & 2.10 & Hydrogen \\
\hline & $161 \mathrm{~A}$ & ASN & 2.85 & Hydrogen \\
\hline & $154 \mathrm{~A}$ & LYS & 5.34 & Salt \\
\hline & $45 \mathrm{~A}$ & ARG & 3.64 & Alkyl \\
\hline & $116 \mathrm{~B}$ & ALA & 3.75 & Alkyl \\
\hline & $155 \mathrm{~B}$ & PRO & 3.67 & Pi-alkyl \\
\hline \multirow{8}{*}{ CID: 442428 Naringin } & $116 \mathrm{~B}$ & ALA & 3.84 & Hydrophobic \\
\hline & $154 \mathrm{~B}$ & LYS & 3.76 & Hydrophobic \\
\hline & $163 \mathrm{~A}$ & GLU & 3.86 & Hydrophobic \\
\hline & $194 \mathrm{~A}$ & THR & 3.75 & Hydrophobic \\
\hline & $120 \mathrm{~B}$ & GLU & 3.22 & Hydrogen \\
\hline & $154 \mathrm{~B}$ & LYS & 3.11 & Hydrogen \\
\hline & $161 \mathrm{~A}$ & ASN & 3.99 & Hydrogen \\
\hline & $228 \mathrm{~B}$ & GLY & 3.04 & Hydrogen \\
\hline \multirow{10}{*}{ CID: 5280805 Rutin } & $45 \mathrm{~A}$ & ARG & 3.64 & Hydrophobic \\
\hline & $163 \mathrm{~A}$ & GLU & 3.59 & Hydrophobic \\
\hline & 194B & THR & 3.71 & Hydrophobic \\
\hline & 196B & TRP & 3.71 & Hydrophobic \\
\hline & $45 \mathrm{~A}$ & ARG & 3.84 & Hydrogen \\
\hline & $116 \mathrm{~B}$ & ALA & 4.03 & Hydrogen \\
\hline & $120 \mathrm{~B}$ & GLU & 3.10 & Hydrogen \\
\hline & $161 \mathrm{~A}$ & ASN & 3.20 & Hydrogen \\
\hline & 197B & TYR & 2.93 & Hydrogen \\
\hline & $224 \mathrm{~B}$ & GLU & 3.44 & Hydrogen \\
\hline \multirow{6}{*}{ CID: 28523 Tomatine } & $134 \mathrm{~A}$ & TYR & 3.97 & Hydrophobic \\
\hline & $166 \mathrm{~A}$ & VAL & 3.75 & Hydrophobic \\
\hline & $32 \mathrm{~B}$ & GLY & 3.42 & Hydrogen \\
\hline & $45 \mathrm{~A}$ & ARG & 3.25 & Hydrogen \\
\hline & $161 \mathrm{~A}$ & ASN & 4.08 & Hydrogen \\
\hline & $230 \mathrm{~B}$ & THR & 3.25 & Hydrogen \\
\hline \multirow{7}{*}{ CID: 447077 Standard MAPK6 inhibitor } & $110 \mathrm{~A}$ & TYR & 3.49 & Hydrophobic \\
\hline & $154 \mathrm{~B}$ & LYS & 3.67 & Hydrophobic \\
\hline & $163 \mathrm{~A}$ & GLU & 3.98 & Hydrophobic \\
\hline & 194B & THR & 3.78 & Hydrophobic \\
\hline & 196B & TRP & 3.73 & Hydrophobic \\
\hline & 197B & TYR & 3.42 & Hydrogen \\
\hline & $224 \mathrm{~B}$ & GLU & 3.88 & Salt bridge \\
\hline
\end{tabular}

Table 4. List of bonding interactions between selected four bioactive compounds and standard MAPK6 inhibitor with MAPK6.

complex (Fig. 13). Binding of TOM decreased the backbone Rg values till $10 \mathrm{~ns}$. In the time period between 11 and $50 \mathrm{~ns}$ there were no considerable fluctuations and almost constant value of $\sim 1.96 \mathrm{~nm}$ was maintained. Till end, the $\mathrm{Rg}$ values were found to be in the range of 1.94-2.00 $\mathrm{nm}$. Complete analysis revealed that, in the initial stage, the trajectory had shown its peak value of $\sim 2.10 \mathrm{~nm}$. Later, this high value was never displayed again which shows the stability of protein in the complex (Fig. 13). The complete interpretation revealed that both the molecules induced no major structural changes in the protein.

Moreover, analysis of Solvent Accessible Surface Area (SASA) for all the complexes was implemented. SASA is the substantial criterion to examine the extent of exposure of receptor to the surrounding solvent molecules during simulation ${ }^{47,51}$. In general, binding of ligand may induce the structural changes in the receptor and hence the area in contact with the solvent also may vary ${ }^{49}$. SASA values of protein was plotted against time to 


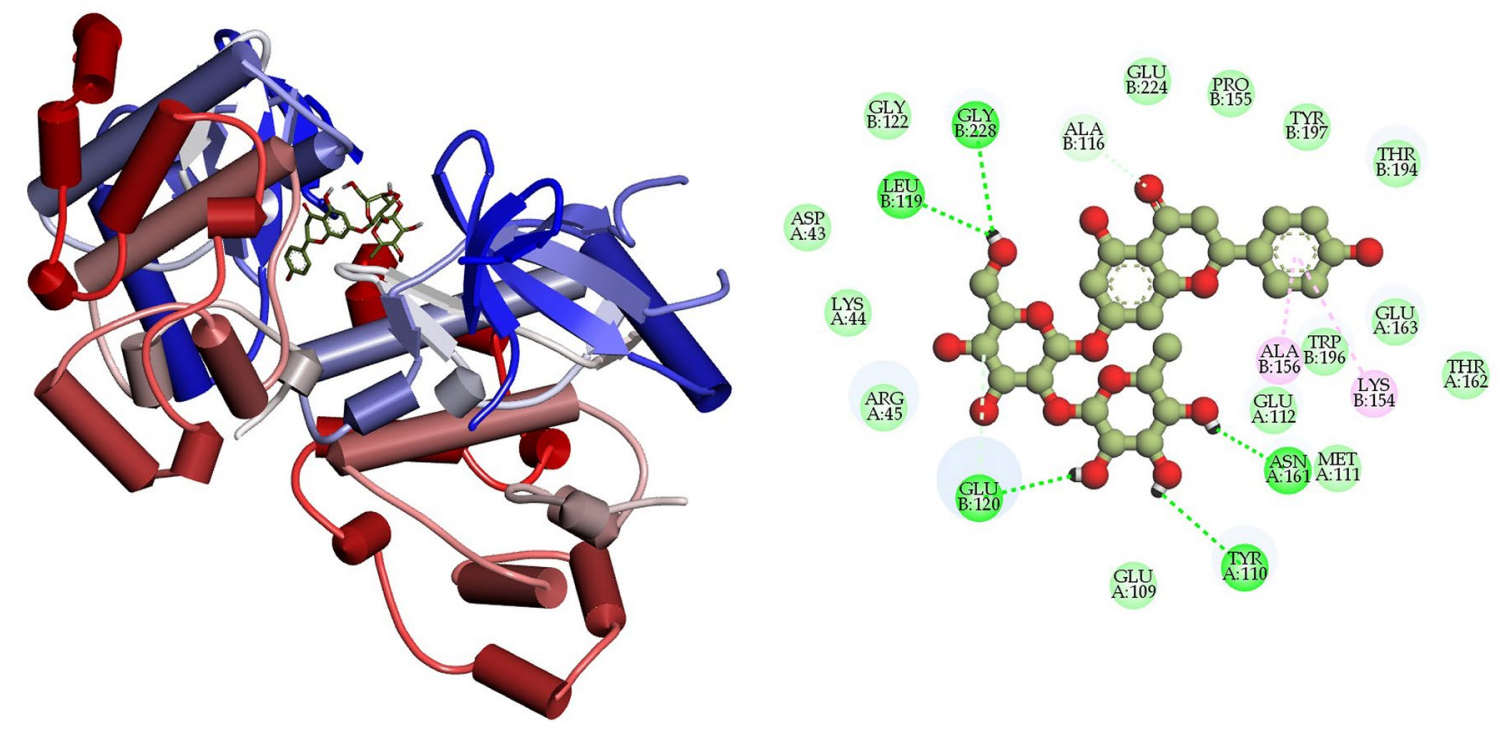

Figure 5. Depicted the interaction between the compound CID: 442428 (Naringin) and MAPK6. Left side representing $3 \mathrm{D}$ and the right side representing $2 \mathrm{D}$ complex protein-ligand interaction.
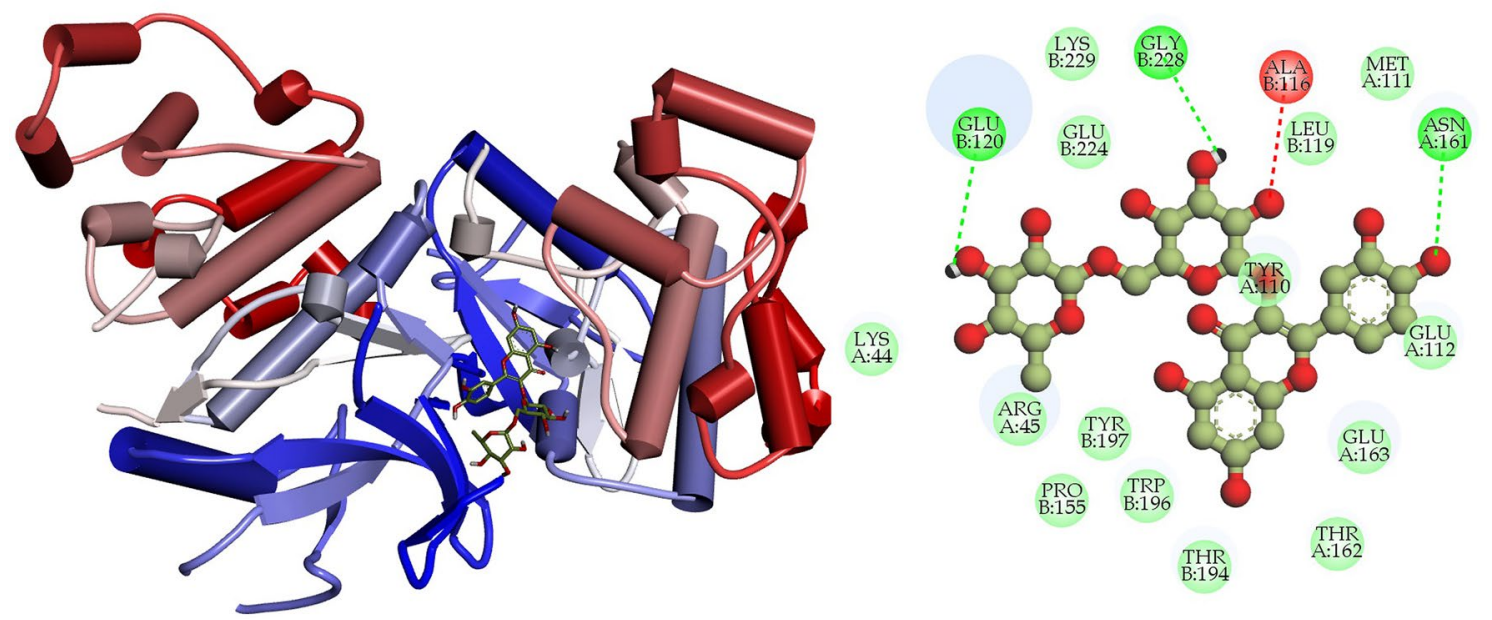

Figure 6. Depicted the interaction between the compound CID: 5280805 (Rutin) and MAPK6. Left side representing $3 \mathrm{D}$ and the right side representing $2 \mathrm{D}$ complex protein-ligand interaction.
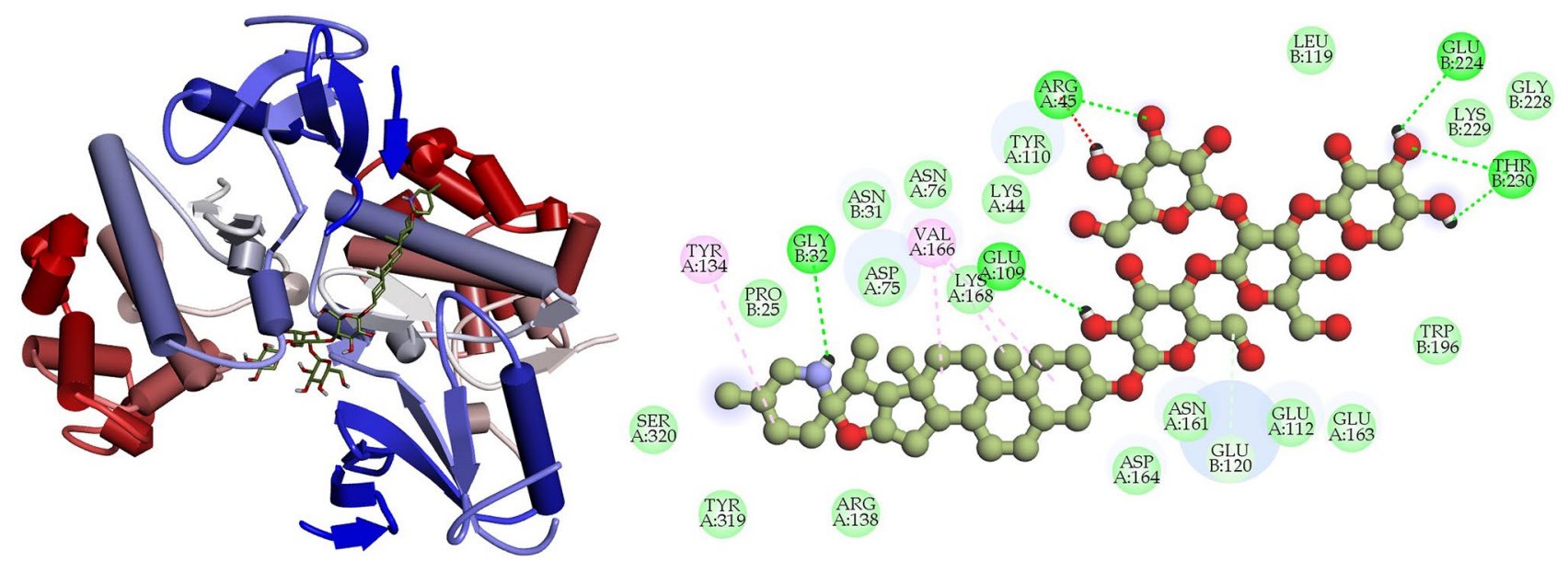

Figure 7. Depicted the interaction between the compound CID: 28523 (Tomatine) and MAPK6. Left side representing $3 \mathrm{D}$ and the right side representing $2 \mathrm{D}$ complex protein-ligand interaction. 


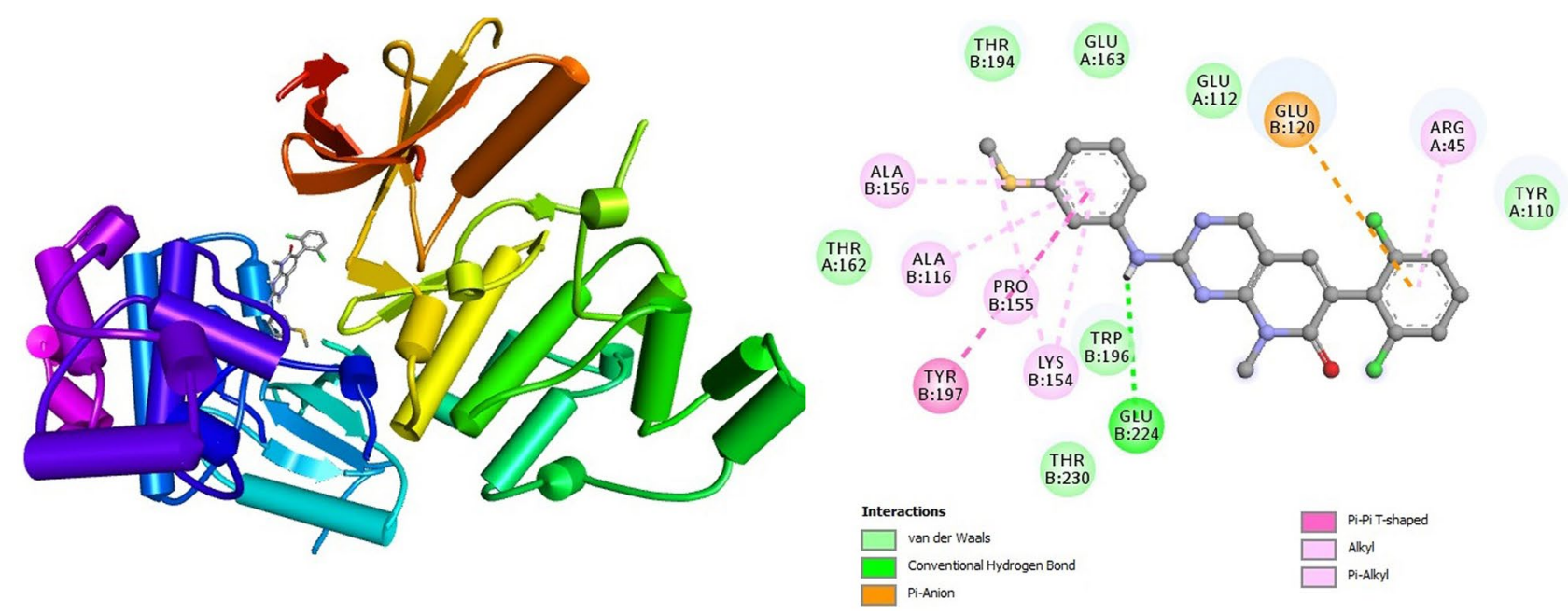

Figure 8. Depicted the interaction between the standard MAPK6 inhibitor (CID: 447077) drug and MAPK6. Left side representing $3 \mathrm{D}$ and the right side representing $2 \mathrm{D}$ complex protein-ligand interaction.

\begin{tabular}{|c|c|c|c|c|c|}
\hline Parameter & CID: 12041593 & CID: 442428 & CID: 5280805 & CID: 28523 & CID: 447077 \\
\hline Formula & $\mathrm{C}_{24} \mathrm{H}_{30} \mathrm{O}_{5}$ & $\mathrm{C}_{27} \mathrm{H}_{32} \mathrm{O}_{14}$ & $\mathrm{C}_{27} \mathrm{H}_{30} \mathrm{O}_{16}$ & $\mathrm{C}_{50} \mathrm{H}_{85} \mathrm{NO}_{19}$ & $\mathrm{C}_{21} \mathrm{H}_{16} \mathrm{C}_{12} \mathrm{~N}_{4} \mathrm{OS}$ \\
\hline $\mathrm{MW}\left(\mathrm{g} \mathrm{mol}^{-1}\right)$ & 398.49 & 580.53 & 610.52 & 1431.59 & 443.35 \\
\hline Num. heavy atoms & 29 & 41 & 43 & 101 & 29 \\
\hline Num. arom. heavy atoms & 10 & 12 & 16 & 12 & 22 \\
\hline Fraction Csp3 & 0.54 & 0.52 & 0.44 & 1 & 0.10 \\
\hline Num. rotatable bonds & 3 & 6 & 6 & 20 & 4 \\
\hline Num. H-bond acceptors & 5 & 14 & 16 & 29 & 21 \\
\hline Num. H-bond donors & 2 & 8 & 10 & 15 & 1 \\
\hline Molar Refractivity & 113.38 & 134.91 & 141.38 & 251.13 & 121.78 \\
\hline TPSA $\left(\AA^{2}\right)$ & 79.90 & 225.06 & 269.43 & 308.40 & 85.11 \\
\hline Solubility class & Moderately soluble & Soluble & Soluble & Moderately soluble & Poorly soluble \\
\hline GI absorption & High & Low & Low & Low & High \\
\hline BBB permeation & No & No & No & No & No \\
\hline Violation of Lipinskis rule of five & 0 & 3 & 3 & 3 & 1 \\
\hline Violation of Veber rule & 0 & 1 & 1 & 1 & 0 \\
\hline Bioavailability Score & 0.55 & 0.17 & 0.17 & 0.17 & 0.55 \\
\hline Synthetic accessibility & 5.16 & 6.16 & 6.52 & 10.00 & 3.07 \\
\hline
\end{tabular}

Table 5. Pharmacokinetics and physicochemical parameters of selected bioactive compounds and standard MAPK6 inhibitor.

estimate the changes in surface area. For SASA complex, the trajectory showed decrease in the values till $15 \mathrm{~ns}$. Except few time intervals, minute fluctuations were observed throughout the simulation period (Fig. 14). The average SASA value was found to be $\sim 138 \mathrm{~nm}^{2}$ and were in the range of $150-130 \mathrm{~nm}^{2}$. For NAR complex, the trajectory showed decrease in the values till $10 \mathrm{~ns}$. Except few time intervals, minute fluctuations were observed throughout the simulation period (Fig. 14). The average SASA value was found to be $\sim 142 \mathrm{~nm}^{2}$ and were in the range of 149-134 $\mathrm{nm}^{2}$. For RUT complex, the trajectory showed decrease in the values till $10 \mathrm{~ns}$. In the time interval of 11-28 ns, minute fluctuations were observed and from 29 to $34 \mathrm{~ns}$ a moderate fluctuation was observed (Fig. 14). The average SASA value was found to be $\sim 140 \mathrm{~nm}^{2}$ and were in the range of $154-133 \mathrm{~nm}^{2}$. For TOM complex, the trajectory showed decrease in the values till $10 \mathrm{~ns}$. Except few time intervals, minute fluctuations were observed throughout the simulation period (Fig. 14). The average SASA value was found to be $\sim 148 \mathrm{~nm}^{2}$ and were in the range of $154-140 \mathrm{~nm}^{2}$. Overall, the analyses revealed that the surface area of protein in complexes were shrunken during the simulation.

To examine the binding energy of the ligands with the target protein, the MD trajectories were analyzed to interpret the extent of hydrogen bond formation during the entire course of simulation and was depicted in Fig. 15. SASA had formed good number of $\mathrm{H}$-bonds with the receptor protein with a maximum of five bonds at several time frames indicating the stronger affinity towards the target. Consistency was maintained in forming almost two hydrogen bonds for the entire simulation time which signifies the stability of the complex. For the 

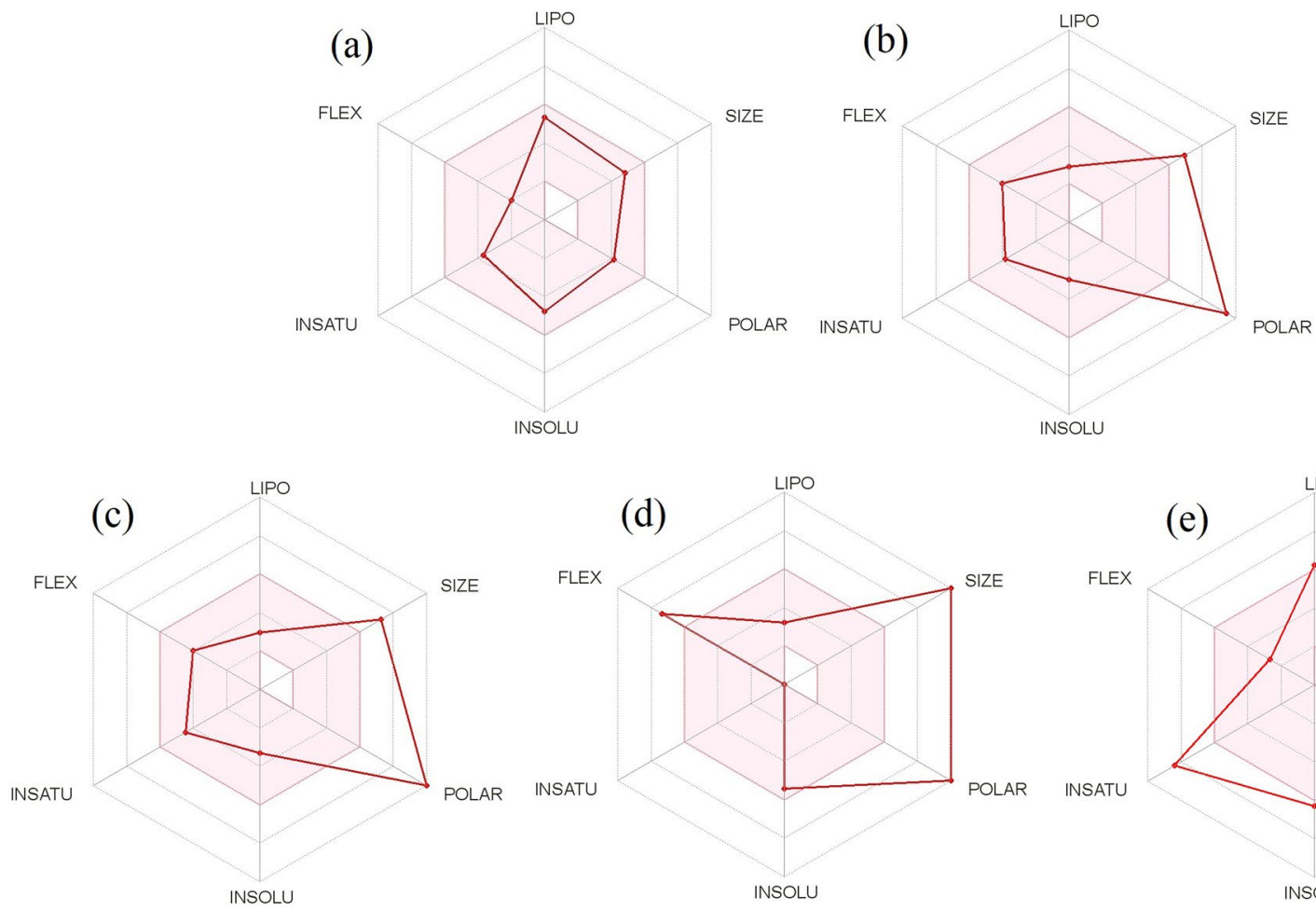

\begin{abstract}
(d)
\end{abstract}
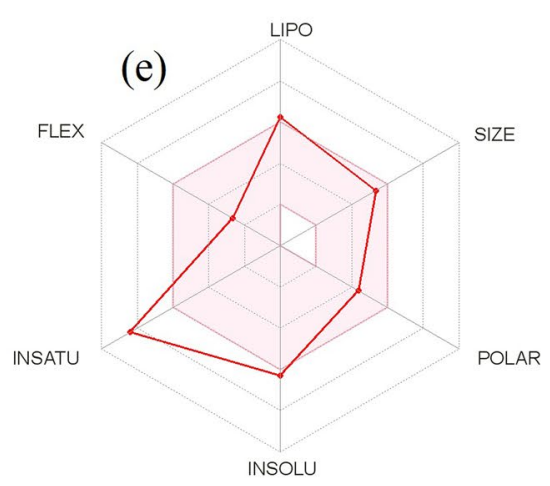

Figure 9. Bioavailability radar plot for oral bioavailability of selected bioactive compounds. CID: 12041593 (ASA) (a), CID: 442428 (NAR) (b), CID: 5280805 (RUT) (c), CID: 28523 (TOM) (d) and CID: 447077 standard MAPK6 inhibitor drug (e). The pink area exhibits the optimal range for each properties (Lipophilicity as XLOGP3 between -0.7 and + 5.0; Size as molecular weight between 150 and $500 \mathrm{~g} \mathrm{~mol}^{-1}$; Polarity as TPSA (topological polar surface area) between 20 and $130 \AA^{2}$; Insolubility in water by log S scale not higher than 6; Insaturation as per fraction of carbons in the sp3 hybridization not less than 0.25 and Flexibility as per rotatable bonds no more than 9).

NAR complex, the consistency was maintained in forming three hydrogen bonds with maximum of six bonds at certain time periods. For RUT complex, the consistency was maintained in forming four hydrogen bonds with maximum of nine bonds at certain time periods. For the TOM complex, the consistency was maintained in forming two hydrogen bonds with maximum of nine bonds at certain time periods. This clearly signifies that the top phytochemicals have the stronger affinity with the target protein.

We also analyzed the binding interaction of 7AQB-ASA, 7AQB-NAR, 7AQB-RUT, 7AQB-TOM and 7AQBSTD complexes at a time interval of $10 \mathrm{~ns}$ (Supplementary 1). For 7AQB-ASA complex ARG70, ILE83 and ASP93 amino acids, for 7AQB-NAR complex LYS67, ARG70 and PHE81 amino acids, for 7AQB-RUT complex HIS61, ARG151 and HIS187 amino acids, for 7AQB-TOM complex LEU33, VAL34 and LYS49 amino acids and for 7AQB-STD complex GLU112 and TRP196 amino acids were found to have constant interaction during the entire period of simulation (Supplementary file 2).

Binding free energy analysis/molecular mechanics Poisson-Boltzmann surface area (MM/ PBSA). For the last $20 \mathrm{~ns}$ (30-50 ns) of simulation trajectories with dt 1000 frames, the binding free energy ( $\triangle$ G bind) was calculated for 7AQB-ASA, 7AQB-NAR, 7AQB-RUT, 7AQB-TOM and 7AQB-STD complexes by utilizing MM/PBSA method. $\triangle \mathrm{G}$ bind of 7AQB-ASA, 7AQB-NAR, 7AQB-RUT, 7AQB-TOM and 7AQB-STD complexes were $-192.310 \pm 26.072 \mathrm{~kJ} \mathrm{~mol}^{-1},-189.432 \pm 25.360 \mathrm{~kJ} \mathrm{~mol}^{-1}, 199.407 \pm 32.354 \mathrm{~kJ} \mathrm{~mol}^{-1},-213.069 \pm$ $28.252 \mathrm{~kJ} \mathrm{~mol}^{-1}$ and $187.134 \pm 33.329 \mathrm{~kJ} \mathrm{~mol}^{-1}$ respectively. These high negative values of free binding energies signify that four test ligands and standard have high binding affinity towards MAPK6.

\title{
Discussion
}

The purpose of this research work was to look at the cancer-preventive impact of bioactive chemicals found in the south Indian cuisine Rasam by using graph theoretical network and pharmacoinformatics analysis. Pharmacoinformatics is a collection of in silico molecular modeling tools for screening the bioactive substances based on their binding affinities, pharmacokinetics, and pharmacodynamic features ${ }^{52}$. By enabling researchers to narrow down the biological and synthetic research impacts, pharmacoinformatics has sped up the discovery of bioactive substances. Several substances have their positive effects predicted using pharmacoinformatics research, which were then validated by in vitro and in vivo activities. Understanding how chemicals bind, interact, and inhibit/ stimulate a certain protein might help researchers find therapeutic options for certain disease conditions. 


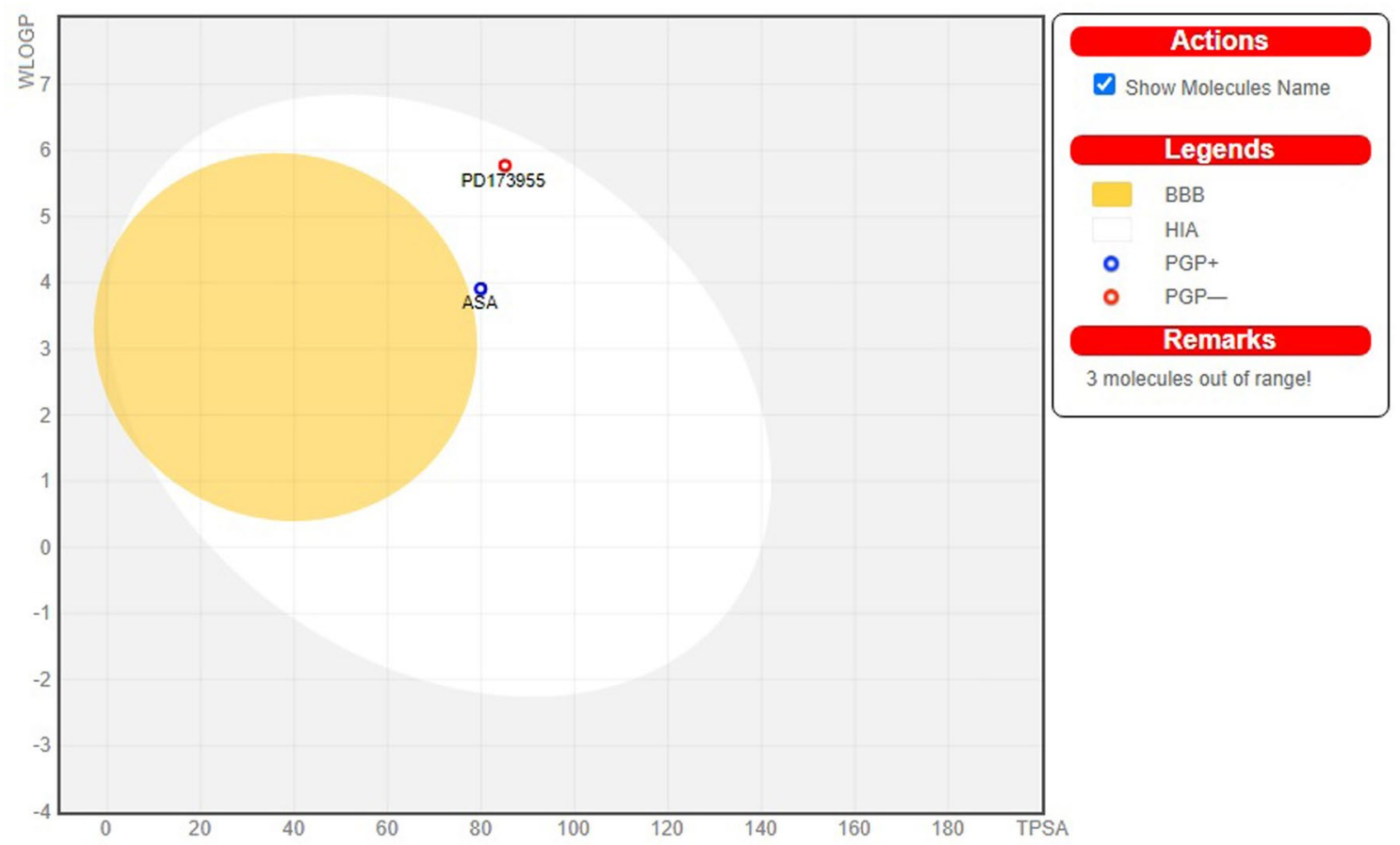

Figure 10. The EGG-BOILED model for the selected bioactive and standard MAPK6 inhibitor drug. The EGG-BOILED represents for intuitive evaluation of passive gastrointestinal absorption (HIA) white part and brain penetration (BBB) yellow part as well as substrates (PGP +) and non-substrates (PGP-) of the permeability glycoprotein (PGP) are represented by blue and red color circles, respectively, of the selected bioactive compound and standard MAPK6 inhibitor in the WLOGP-versus-TPSA graph. The grey region is the physicochemical space of compounds predicted to exhibit high intestinal absorption.

\begin{tabular}{|l|l|l|l|l|l|l|l|l|l|}
\hline Compound ID & AMES toxicity & $\begin{array}{l}\text { Max. tolerated } \\
\text { dose (human) }\end{array}$ & hERG inhibition & LD50 & Hepatotoxicity & Carcinogenicity & $\begin{array}{l}\text { Skin } \\
\text { Sensitisation }\end{array}$ & $\begin{array}{l}\text { T. pyriformis } \\
\text { toxicity }\end{array}$ & $\begin{array}{l}\text { Minnow } \\
\text { toxicity }\end{array}$ \\
\hline $\begin{array}{l}\text { CID: } 12041593 \\
\text { Assafoetidinol A }\end{array}$ & No & -0.448 & No & 2.889 & Yes & No & No & 0.651 \\
\hline $\begin{array}{l}\text { CID: } 442428 \\
\text { Naringin }\end{array}$ & Yes & 0.491 & No & 2.652 & No & No & No & 0.285 \\
\hline $\begin{array}{l}\text { CID: } 5280805 \\
\text { Rutin }\end{array}$ & No & 0.435 & No & 2.472 & No & No & No & 0.285 \\
\hline $\begin{array}{l}\text { CID: } 28523 \\
\text { Tomatine }\end{array}$ & No & 0.216 & No & 2.482 & No & No & No & 0.285 \\
\hline $\begin{array}{l}\text { CID: } 447077 \\
\text { PD- } 173955\end{array}$ & No & 0.205 & No & 2.801 & Yes & No & No & 0.299 \\
\hline
\end{tabular}

Table 6. List of the drug-induced hERG inhibition, AMES toxicity, carcinogens, Tetrahymena pyriformis (TP) toxicity, rat acute toxicity $\left(\mathrm{LD}_{50}\right.$ in $\left.\mathrm{mol} / \mathrm{kg}\right)$, and skin sensitisation along with Minnow toxicity of selected four compounds.

Initially, a graph theoretical network was developed using centrality metrics, and it was suggested for metabolic networks that included enzymatic cascades and synergistic ligand-enzyme interactions. Biological networks, which are made up of a number of vertices (or nodes) linked in a pattern by a set of edges (or connections), are designed to mimic the structure of genuine biological systems. MAPK6/ERK3 was identified as a receptor (target) for ligand (bioactive substances) binding in ROS-induced oxidative stress that leads to malignancies, according to the network analysis study. MAPK6 pathway has been shown to be impacted not just by receptor ligand interactions, but also by various other stresses. MAPK6 is shown to be a key factor for organogenesis, cancer cell growth and invasiveness. MAPK6 overexpression has been detected in numerous human cancers, including squamous cell lung carcinoma, oral squamous cell carcinoma, gastric cancer, breast cancer and melanoma ${ }^{53}$. DNA microarray studies have produced inconsistent information about the regulation of MAPK6 expression in cancer. Several investigations demonstrated that expression of MAPK6 mRNA is down-regulated in brain tumors, ovarian carcinoma and cutaneous melanoma, and up-regulated in leukemias, adrenocortical carcinoma, squamous cell lung carcinoma, salivary adenoid cystic carcinoma, tongue squamous cell carcinoma and cervical cancer ${ }^{54}$. Furthermore, since MAPK signaling cascade regulate both mitogen- and stress-activated signals, the regulation of both pathways by ROS has drawn researchers' attention ${ }^{55}$. The goal of the current research work was to look 


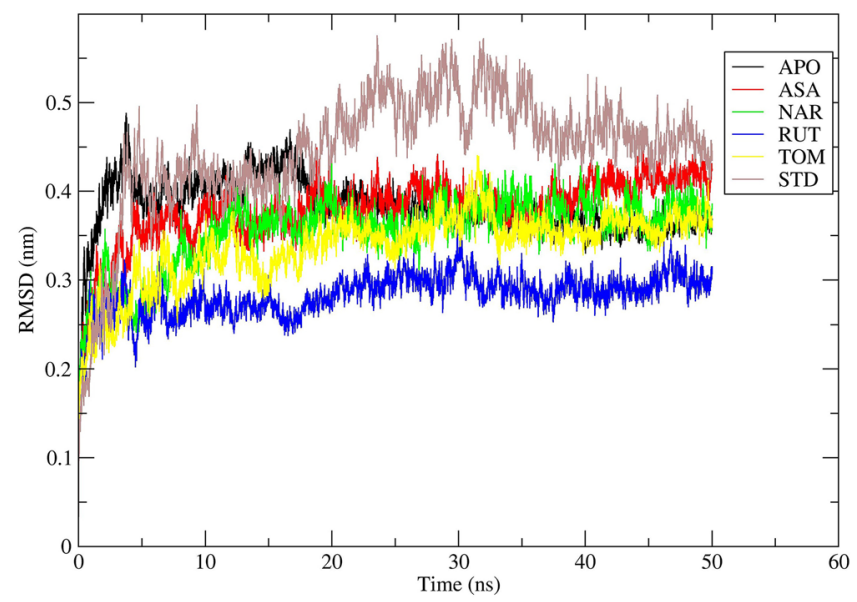

Figure 11. RMSD study plot for $50 \mathrm{~ns}$ MD Simulation of 7AQB-APO (Black), 7AQB-ASA (Red), 7AQB-NAR (Green), 7AQB-RUT (Blue), 7AQB-TOM (Yellow) and 7AQB-STD (Brown).

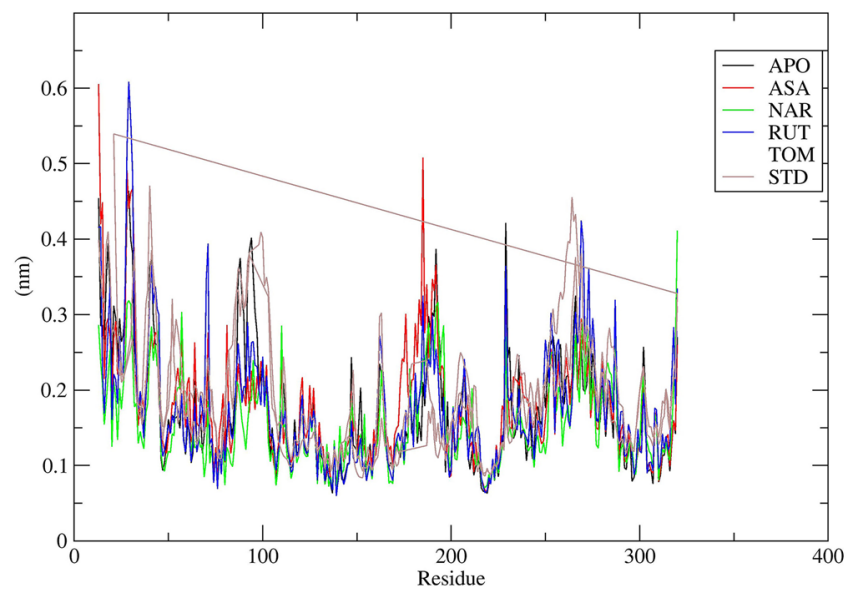

Figure 12. RMSF study plots for $50 \mathrm{~ns} M D$ Simulation. Chain of 7AQB-APO (Black), 7AQB-ASA (Red), 7AQB-NAR (Green), 7AQB-RUT (Blue7AQB-TOM (Yellow) and 7AQB-STD (Brown).

into the detoxification/neutralization of ROS by employing bioactive chemicals found in Rasam spices to protect cells against cancer. A total of sixty-six bioactive compounds were chosen from twelve spices using the IMPPAT database, as well as previously published publications on their effects against different human malignancies. All the chemicals chosen were docked against MAPK6 protein kinases, with binding energy ranging from $-3.50 \mathrm{kcal} /$ $\mathrm{mol}^{-1}$ to $-10.60 \mathrm{kcal} \mathrm{mol}^{-1}$. Four compounds (Assafoetidinol A $\left(-9.80 \mathrm{kcal} \mathrm{mol}^{-1}\right)$, Naringin $\left(-9.60 \mathrm{kcal} \mathrm{mol}^{-1}\right)$, Rutin $\left(-9.80 \mathrm{kcal} \mathrm{mol}^{-1}\right)$, Tomatine $\left.\left(-10.60 \mathrm{kcal} \mathrm{mol}^{-1}\right)\right)$ and PD-173955 (CID: 447077) (-9.30 kcal mol $\left.{ }^{-1}\right)$ have been chosen for future analysis based on their significant binding ability, strong hydrophobic and hydrogen bonding interactions with amino acid residues present in the active site of MAPK6 protein. Similarly, Ahammad et al. identified seventy phytochemicals from Neem (Azadiractha indica) plant, and screened against MCM7 protein by using the molecular docking simulation tool. The binding affinities found after molecular docking of the phytochemicals compound have shown a distributed range between -3.1 and -9.0 and kcal mol ${ }^{-156}$.

Bioactivity of a substance is largely governed by its absorption, distribution, metabolism, and excretion (ADME) characteristics, all of which are connected to its pharmacokinetic characteristics. The bioavailability of dietary phytochemicals to target cells, as well as their absorption and metabolism in the human body are certain key aspects in promoting their bioactivity and maintaining body health ${ }^{57}$. The small intestine absorbs some, but not all, of the components of dietary phytochemicals into the circulatory system. Some phytochemical compounds that were absorbed by the colon and altered by the gut microbiota; the microbial metabolites that were released back into the circulation showed significant activity ${ }^{58}$. In order for any molecule to permeate the membrane, phytochemicals/test substance must break hydrogen bonds in the aqueous environment and partition across the membrane ${ }^{59}$. The polar surface area (PSA) of a chemical is connected to its hydrogen-bonding potential, whereas molecular mass and lipophilicity are associated to membrane permeability ${ }^{60}$. As a consequence, the ADME properties must be assessed at the earlier stages of drug design and discovery process in order to pass the standard clinical studies required to be considered as prospective therapeutic candidate ${ }^{61}$. In this study, all the discovered phytoconstituents were confirmed in terms of usual pharmacokinetic properties using multiple 


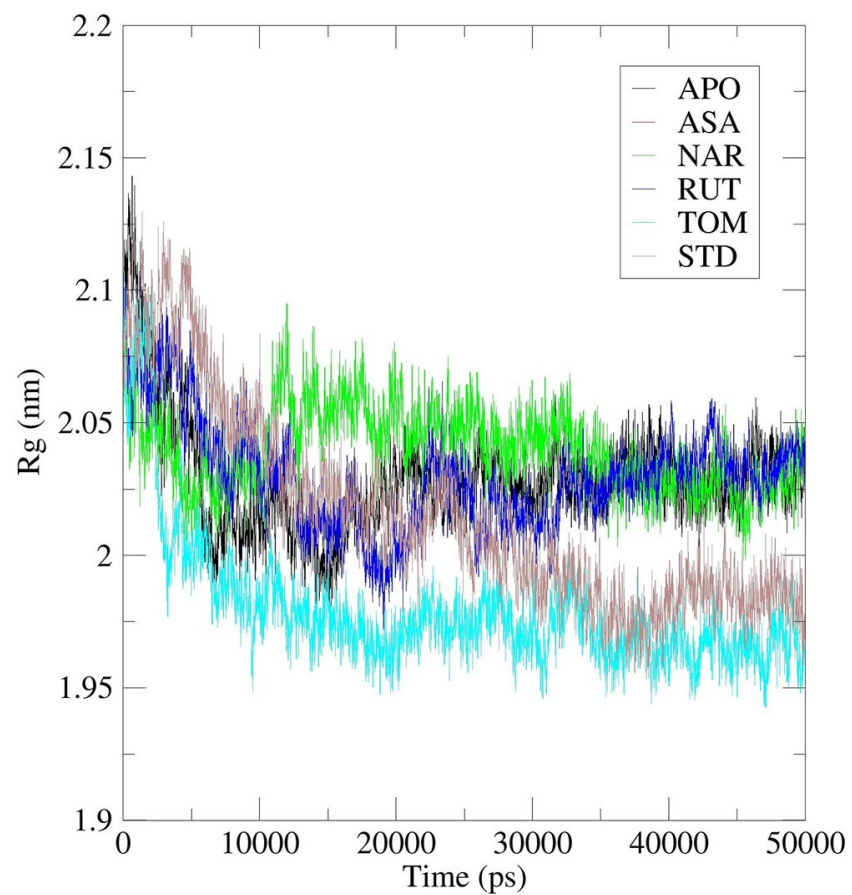

Figure 13. Radius of gyration study plot for $50 \mathrm{~ns}$ MD Simulation of 7AQB-APO (Black), 7AQB-ASA (Red), 7AQB-NAR (Green), 7AQB-RUT (Blue) 7AQB-TOM (Yellow) and 7AQB-STD (Brown).

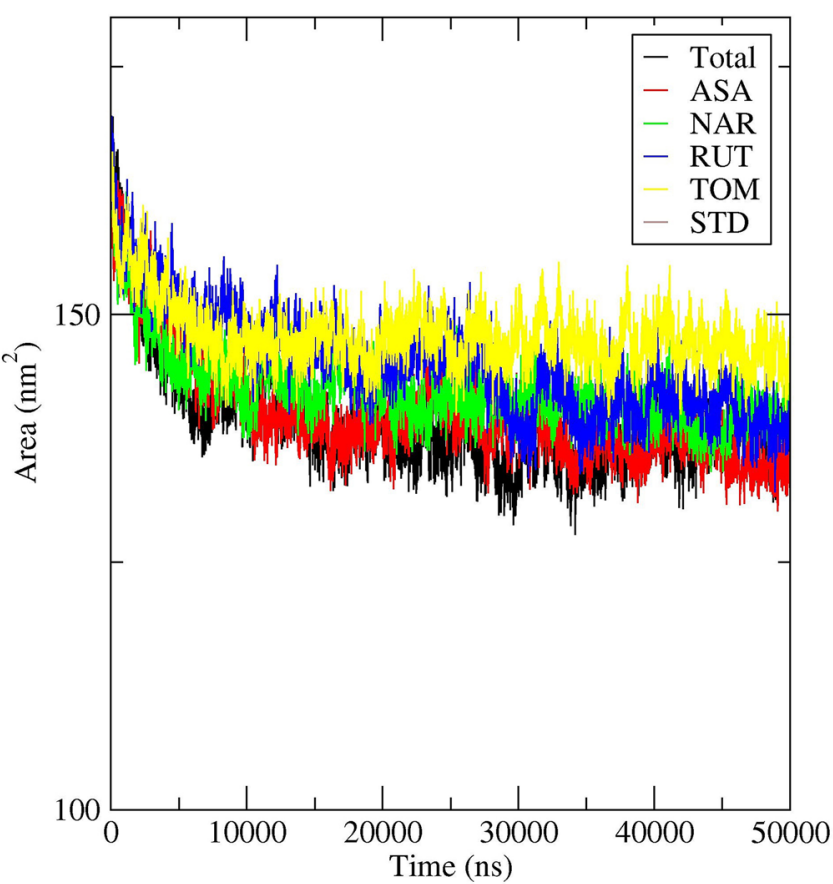

Figure 14. Solvent accessible surface area study plot for $50 \mathrm{~ns}$ MD Simulation of 7AQB-APO (Black), 7AQBASA (Red), 7AQB-NAR (Green), 7AQB-RUT (Blue) 7AQB-TOM (Yellow) and 7AQB-STD (Brown).

bioinformatics methods. Phytochemicals are naturally derived from variety of plants that are often consumed by humans and are usually considered safe to consume. While most phytochemicals are not regulated by the Food and Drug Administration (FDA) in the United States, their potential toxicity is unknown.

Phytochemicals are utilized as supplements in conjunction with illness therapy all around the world, but the users do not necessarily inform this to their physicians ${ }^{62}$. Substance toxicity refers to the property of any compound to be poisonous and to cause harm to an organism. Toxicity testing of a substance necessitates in vitro 


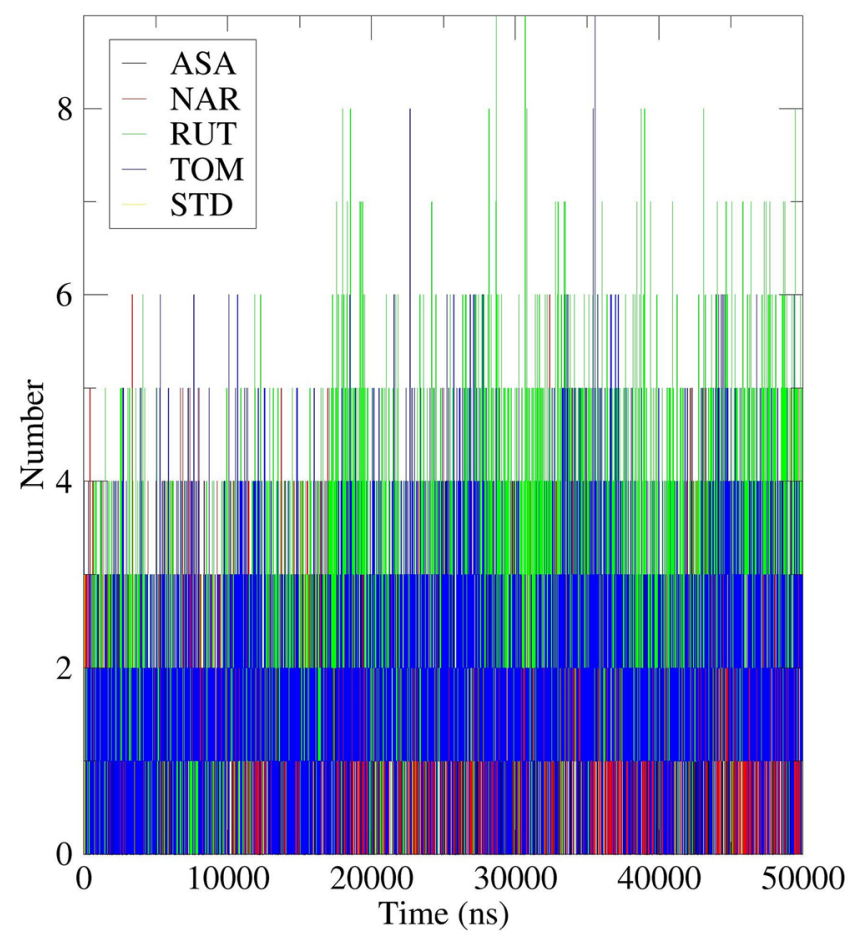

Figure 15. Intermolecular hydrogen bonding study plot for $50 \mathrm{~ns}$ MD Simulation of 7AQB-APO (Black), 7AQB-ASA (Red), 7AQB-NAR (Green), 7AQB-RUT (Blue) 7AQB-TOM (Yellow) and 7AQB-STD (Brown).

and in vivo animal experiments, which is a time-consuming, expensive, and complicated technique. Because there are no animal trials, and improved precision, accessibility, and speed, in silico toxicity assessment has become very popular in recent times and it can offer information on any synthetic or natural molecule. In this work, in silico approaches were used to estimate the toxicity levels of four chemicals. The non-carcinogenic and non-skin irritating properties of four substances were determined using in silico testing. Three compounds, Assafoetidinol A, Rutin, and Tomatine were shown to be negative in Ames testing. Toxicity tests revealed that the four phytochemicals chosen had no negative side effects ( $h E R G$ ). The $\mathrm{LD}_{50}$ (median fatal dosage) indicates the immediate or acute toxicity of substances that were determined to be the most effective in the investigation. Hence, the complexes of these compounds were subjected to molecular dynamics simulations and the results were analysed with the results of apo form of MAPK6 (7AQB). The complexes were validated by interpreting the RMSD, RMSF, Rg, SASA and the lead phytochemical complexes were found to be stable during the simulations.

\section{Conclusion}

Traditionally, home-cooked meals have been shown to help avoid chronic illnesses, improve health, and save treatment costs while also boosting quality of life. This study looked at the antioxidant properties of bioactive chemicals found in the south Indian cuisine, Rasam against oxidative stress-induced human malignancies. In the human body, ROS is a metabolic by-product of cellular respiration. Oxidative stress and overexpression of MAPK6 protein are caused by an increase in ROS levels. MAPK6 overexpression causes a cascade of events in cells, including mutations and carcinogenesis. Through a thorough pharmacoinformatics-based molecular docking investigation of bioactive substances against MAPK6, the antioxidant potential of Rasam has been proven in the current work. In silico molecular docking investigations found that the four lead phytochemicals (Assafoetidinol A, Naringin, Rutin, and Tomatine) may suppress MAPK6/ERK3 expression. In addition, MD stimulation tests and in silico pharmacokinetic prediction analyses gives the safety profile of four lead compounds as well as the stability of the protein-ligand complex, although, in order to determine the Rasam's effectiveness, further in vitro and in vivo animal research work will be necessary.

Received: 27 June 2021; Accepted: 5 October 2021

Published online: 02 November 2021

\section{References}

1. Kunjiappan, S. et al. Capsaicin-loaded solid lipid nanoparticles: Design, biodistribution, in silico modeling and in vitro cytotoxicity evaluation. Nanotechnology 32, 095101 (2020).

2. Sung, H. et al. Global cancer statistics 2020: GLOBOCAN estimates of incidence and mortality worldwide for 36 cancers in 185 countries. CA Cancer J. Clin. 71, 209-249 (2021). 
3. Kushi, L. H. et al. American Cancer Society Guidelines on nutrition and physical activity for cancer prevention: reducing the risk of cancer with healthy food choices and physical activity. CA Cancer J. Clin. 62, 30-67 (2012).

4. Perše, M. The role of the antioxidant defense system in the pathogenesis of colorectal cancer. Free Radic. 217 (Nova Science Publisher Inc. 2014).

5. Ren, J., Sowers, J. R. \& Zhang, Y. Autophagy and Cardiometabolic Diseases: From Molecular Mechanisms to Translational Medicine (Academic Press, 2018).

6. Kunjiappan, S. et al. Design, in silico modelling and functionality theory of folate-receptor-targeted myricetin-loaded bovine serum albumin nanoparticle formulation for cancer treatment. Nanotechnology 31, 155102 (2020).

7. Palmeira, C. M. et al. Mitohormesis and metabolic health: The interplay between ROS, cAMP and sirtuins. Free Radic. Biol. Med. 141, 483-491 (2019).

8. Nordberg, J. \& Arnér, E. S. Reactive oxygen species, antioxidants, and the mammalian thioredoxin system. Free Radic. Biol. Med. 31, 1287-1312 (2001).

9. Ganesan, V. et al. Optimization and analysis of microwave-assisted extraction of bioactive compounds from Mimosa pudica L. using RSM \& ANFIS modeling. J. Food Meas. Charact. 12, 228-242 (2018).

10. Sachdev, S., Ansari, S. A., Ansari, M. I., Fujita, M. \& Hasanuzzaman, M. Abiotic stress and reactive oxygen species: Generation, signaling, and defense mechanisms. Antioxidants 10, 277 (2021).

11. Mates, J. Effects of antioxidant enzymes in the molecular control of reactive oxygen species toxicology. Toxicology 153, 83-104 (2000).

12. Xiao, H., Lü, F., Stewart, D. \& Zhang, Y. Mechanisms underlying chemopreventive effects of flavonoids via multiple signaling nodes within Nrf2-ARE and AhR-XRE gene regulatory networks. Curr. Chem. Biol. 7, 151-176 (2013).

13. Roux, P. P. \& Blenis, J. ERK and p38 MAPK-activated protein kinases: A family of protein kinases with diverse biological functions. Microbiol. Mol. Biol. Rev. 68, 320-344 (2004).

14. Cargnello, M. \& Roux, P. P. Activation and function of the MAPKs and their substrates, the MAPK-activated protein kinases. Microbiol. Mol. Biol. Rev. 75, 50-83 (2011).

15. Alsaran, H. M. Functional characterization of cancer-related mutations of ERK3. (2016).

16. Bogucka, K. et al. ERK3/MAPK6 controls IL-8 production and chemotaxis. Elife 9, e52511 (2020).

17. Long, W. et al. ERK3 signals through SRC-3 coactivator to promote human lung cancer cell invasion. J. Clin. Investig. 122, $1869-1880$ (2012).

18. Sachan, A., Kumar, S., Kumari, K. \& Singh, D. Medicinal uses of spices used in our traditional culture: Worldwide. J. Med. Plants Stud. 6, 116-122 (2018).

19. Zheng, J. et al. Spices for prevention and treatment of cancers. Nutrients 8, 495 (2016).

20. Tamvakopoulos, C. et al. Metabolism and anticancer activity of the curcumin analogue, dimethoxycurcumin. Clin. Cancer Res. 13, 1269-1277 (2007).

21. Raza, K. et al. Conjugation of docetaxel with multiwalled carbon nanotubes and codelivery with piperine: Implications on pharmacokinetic profile and anticancer activity. Mol. Pharm. 13, 2423-2432 (2016).

22. Sharoni, Y., Danilenko, M. \& Levy, J. Molecular mechanisms for the anticancer activity of the carotenoid lycopene. Drug Dev. Res. 50, 448-456 (2000).

23. Mohan, U. P. et al. Utilization of plant-derived Myricetin molecule coupled with ultrasound for the synthesis of gold nanoparticles against breast cancer. Naunyn Schmiedebergs Arch. Pharmacol. 393, 1963-1976 (2020).

24. Pandian, S. R. K. et al. Formulation and evaluation of rutin-loaded solid lipid nanoparticles for the treatment of brain tumor. Naunyn Schmiedebergs Arch. Pharmacol. 394, 735-749 (2021).

25. Sheen, B. Foods of India (Greenhaven Publishing LLC, 2006).

26. Devarajan, A. \& Mohanmarugaraja, M. A comprehensive review on Rasam: A South Indian traditional functional food. Pharmacogn. Rev. 11, 73 (2017).

27. Bélanger, J. Green Leafy Vegetables of Rural India: Ethnobotany and, McGill University, (2010).

28. Wang, S., Meckling, K. A., Marcone, M. F., Kakuda, Y. \& Tsao, R. Synergistic, additive, and antagonistic effects of food mixtures on total antioxidant capacities. J. Agric. Food Chem. 59, 960-968 (2011).

29. Pan, M.-H. \& Ho, C.-T. Chemopreventive effects of natural dietary compounds on cancer development. Chem. Soc. Rev. 37, 2558-2574 (2008)

30. Dreosti, I. E. Bioactive ingredients: Antioxidants and polyphenols in tea. Nutr. Rev. 54, S51-S58 (1996).

31. Kunjiappan, S. et al. Design graph theoretical analysis and in silico modeling of dunaliella bardawil biomass encapsulated $\mathrm{N}$-succinyl chitosan nanoparticles for enhanced anticancer activity. Anti-Cancer Agents Med. Chem. Former. Curr. Med. Chem. Anti-Cancer Agents 18, 1900-1918 (2018).

32. Berman, H. M. et al. The protein data bank. Nucleic Acids Res. 28, 235-242 (2000).

33. Mohanraj, K. et al. IMPPAT: A curated database of Indian medicinal plants, phytochemistry and therapeutics. Sci Rep. 8, 4329 (2018).

34. Dallakyan, S. \& Olson, A. J. in Chemical Biology 243-250 (Springer, 2015).

35. Gaillard, T. Evaluation of AutoDock and AutoDock Vina on the CASF-2013 benchmark. J. Chem. Inf. Model. 58, 1697-1706 (2018).

36. Mohan, U. P., Kunjiappan, S., Pichiah, P. T. \& Arunachalam, S. Adriamycin inhibits glycolysis through downregulation of key enzymes in Saccharomyces cerevisiae. 3 Biotech 11, 1-13 (2021).

37. Wang, Y. et al. In silico prediction of human intravenous pharmacokinetic parameters with improved accuracy. J. Chem. Inf. Model. 59, 3968-3980 (2019).

38. Islam, M. A. \& Pillay, T. S. Identification of promising anti-DNA gyrase antibacterial compounds using de novo design, molecular docking and molecular dynamics studies. J. Biomol. Struct. Dyn. 38, 1798-1809 (2020).

39. Jia, C.-Y., Li, J.-Y., Hao, G.-F. \& Yang, G.-F. A drug-likeness toolbox facilitates ADMET study in drug discovery. Drug Discov. Today 25, 248-258 (2020).

40. Markov, S., Petkov, P. \& Pavlov, V. in International Conference on Variability of the Sun and Sun-Like Stars: From Asteroseismology to Space Weather. 359-367 (Springer).

41. Zai, Y. et al. Aggregation and its influence on the bioactivities of a novel antimicrobial peptide, temporin-PF, and its analogues. Int. J. Mol. Sci. 22, 4509 (2021).

42. Rajendran, B. K. et al. Pharmacoinformatic approach to explore the antidote potential of phytochemicals on bungarotoxin from Indian Krait, Bungarus caeruleus. Comput. Struct. Biotechnol. J. 16, 450-461 (2018).

43. Van Aalten, D. M. et al. PRODRG, a program for generating molecular topologies and unique molecular descriptors from coordinates of small molecules. J. Comput. Aided Mol. Des. 10, 255-262 (1996).

44. Kumari, R., Kumar, R., Consortium, O. S. D. D. \& Lynn, A. g_mmpbsa-A GROMACS tool for high-throughput MM-PBSA calculations. J. Chem. Inf. Model. 54, 1951-1962 (2014).

45. Wisniewski, D. et al. Characterization of potent inhibitors of the Bcr-Abl and the c-kit receptor tyrosine kinases. Can. Res. 62, 4244-4255 (2002).

46. Sharma, A., Datta, D. \& Balasubramaniam, R. Molecular dynamics simulation to investigate the orientation effects on nanoscale cutting of single crystal copper. Comput. Mater. Sci. 153, 241-250 (2018). 
47. Kumar, B., Parasuraman, P., Murthy, T. P. K., Murahari, M. \& Chandramohan, V. In silico screening of therapeutic potentials from Strychnos nux-vomica against the dimeric main protease (Mpro) structure of SARS-CoV-2. J. Biomol. Struct. Dyn., 1-19 (2021).

48. Abdelrheem, D. A. et al. The inhibitory effect of some natural bioactive compounds against SARS-CoV-2 main protease: Insights from molecular docking analysis and molecular dynamic simulation. J. Environ. Sci. Health Part A 55, 1373-1386 (2020).

49. Rahman, M. M. et al. Virtual screening, molecular dynamics and structure-activity relationship studies to identify potent approved drugs for Covid-19 treatment. J. Biomol. Struct. Dyn. 39, 6231-6241 (2021).

50. Gupta, S. et al. Identification of potential natural inhibitors of SARS-CoV2 main protease by molecular docking and simulation studies. J. Biomol. Struct. Dyn. 39, 4334-4345 (2021).

51. Ghosh, S. K., Saha, B. \& Banerjee, R. Insight into the sequence-structure relationship of TLR cytoplasm's Toll/Interleukin-1 receptor domain towards understanding the conserved functionality of TLR 2 heterodimer in mammals. J. Biomol. Struct. Dyn. 39, 5348-5357 (2021).

52. Alamri, M. A. Pharmacoinformatics and molecular dynamic simulation studies to identify potential small-molecule inhibitors of WNK-SPAK/OSR1 signaling that mimic the RFQV motifs of WNK kinases. Arab. J. Chem. 13, 5107-5117 (2020).

53. Alshammari, E. S. et al. ERK3 is transcriptionally upregulated by $\Delta \mathrm{Np} 63 \alpha$ and mediates the role of $\Delta \mathrm{Np} 63 \alpha$ in suppressing cell migration in non-melanoma skin cancers. BMC Cancer 21, 1-12 (2021).

54. Meloche, S. MAPK6 (mitogen-activated protein kinase 6). In Atlas of Genetics and Cytogenetics in Oncology and Haematology (2009).

55. Yang, K.-Y., Liu, Y. \& Zhang, S. Activation of a mitogen-activated protein kinase pathway is involved in disease resistance in tobacco. Proc. Natl. Acad. Sci. 98, 741-746 (2001).

56. Ahammad, F. et al. Pharmacoinformatics and molecular dynamics simulation-based phytochemical screening of neem plant (Azadiractha indica) against human cancer by targeting MCM7 protein. Brief. Bioinform. (2021).

57. da Silva, B. V., Barreira, J. C. \& Oliveira, M. B. P. Natural phytochemicals and probiotics as bioactive ingredients for functional foods: Extraction, biochemistry and protected-delivery technologies. Trends Food Sci. Technol. 50, 144-158 (2016).

58. Giampieri, F. et al. The strawberry: Composition, nutritional quality, and impact on human health. Nutrition 28, 9-19 (2012)

59. Selby-Pham, S. N., Miller, R. B., Howell, K., Dunshea, F. \& Bennett, L. E. Physicochemical properties of dietary phytochemicals can predict their passive absorption in the human small intestine. Sci. Rep. 7, 1-15 (2017).

60. Alex, A., Millan, D. S., Perez, M., Wakenhut, F. \& Whitlock, G. A. Intramolecular hydrogen bonding to improve membrane permeability and absorption in beyond rule of five chemical space. MedChem Comm 2, 669-674 (2011).

61. Hughes, J. P., Rees, S., Kalindjian, S. B. \& Philpott, K. L. Principles of early drug discovery. Br. J. Pharmacol. 162, 1239-1249 (2011).

62. Sen, S. \& Chakraborty, R. Revival, modernization and integration of Indian traditional herbal medicine in clinical practice: Importance, challenges and future. J. Tradit. Complement. Med. 7, 234-244 (2017).

\section{Acknowledgements}

The authors are thankful to the KEGG pathway database and Kanehisa Laboratories, Kyoto University, Japan.

\section{Author contributions}

A.K., T.P., P.P., S.R., D.A., S.M., S.K. (8th Author), S.A. and S.K. (10th Author) have done all laboratory works and prepared manuscript and related works. S.M., S.A., K.S. and S.K. (10th Author) has reviewed and made corrections for the manuscript. All authors reviewed the manuscript.

\section{Competing interests}

The authors declare no competing interests.

\section{Additional information}

Supplementary Information The online version contains supplementary material available at https://doi.org/ 10.1038/s41598-021-01008-9.

Correspondence and requests for materials should be addressed to S.A. or S.K.

Reprints and permissions information is available at www.nature.com/reprints.

Publisher's note Springer Nature remains neutral with regard to jurisdictional claims in published maps and institutional affiliations.

(c) (i) Open Access This article is licensed under a Creative Commons Attribution 4.0 International License, which permits use, sharing, adaptation, distribution and reproduction in any medium or format, as long as you give appropriate credit to the original author(s) and the source, provide a link to the Creative Commons licence, and indicate if changes were made. The images or other third party material in this article are included in the article's Creative Commons licence, unless indicated otherwise in a credit line to the material. If material is not included in the article's Creative Commons licence and your intended use is not permitted by statutory regulation or exceeds the permitted use, you will need to obtain permission directly from the copyright holder. To view a copy of this licence, visit http://creativecommons.org/licenses/by/4.0/.

(c) The Author(s) 2021 